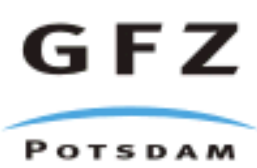

Originally published as:

Popov, A. A., Sobolev, S. V. (2008): SLIM3D: A tool for the three-dimensional thermomechanical modeling of the lithospheric deformation with elasto-visco-plastic rheology. - Physics of the Earth and Planetary Interiors, 171, 1-4, 55-75

DOI: 10.1016/j.pepi.2008.03.007 


\title{
SLIM3D: A tool for three-dimensional thermomechanical modeling of lithospheric deformation with elasto-visco-plastic rheology
}

\author{
A.A. Popov ${ }^{*}$, S.V. Sobolev \\ GeoForschungsZentrum, Telegrafenberg, 14473 Potsdam, Germany
}

\begin{abstract}
We describe a new technique for three-dimensional lithospheric-scale modeling of solid state deformation including strain localization processes. The new code, SLIM3D, includes a coupled thermo-mechanical treatment of deformation processes and allows for an elasto-visco-plastic rheology with diffusion, dislocation and Peierls creep mechanisms and Mohr-Coulomb plasticity. The code incorporates an Arbitrary Lagrangian Eulerian formulation with free surface and Winkler boundary conditions. SLIM3D is developed and implemented using the C++ objectoriented programming language. We describe aspects of physical models as well as details of the numerical implementation, including the Newton-Raphson solver, the stress update procedure, and the tangent operator. The applicability of the code to lithospheric-scale modeling is demonstrated by a number of benchmark problems that include: (i) the bending of an elastic plate, (ii) the sinking of a rigid cylinder into a viscous fluid, (iii) the initiation of shear bands in the brittle crust, (iv) triaxial compression test, and (v) lithospheric transpressional deformation. Finally, we discuss possible directions of further development.
\end{abstract}

Keywords: 3D numerical modeling; Geodynamics; Lithospheric deformation; Strain localization; Mohr-Coulomb

\section{Introduction}

On geological time scales, lithospheric rocks deform by three fundamentally different phenomenological mechanisms. These are: (i) elastic mechanism (reversible), which on geological time-scales occur mostly during flexural deformation of the lithospheric plates or bending of slabs in subduction zones; (ii) viscous mechanism, typical of the convective mantle, or for lower crustal flow at plate boundaries; and (iii) plastic mechanism, which simulate brittle failure and manifests itself in the formation of narrow shear zones or faults.

The quantitative proportion between each of these mechanisms depends on many parameters, the most important of which are temperature and stress. Viscosity, for instance, varies by several orders of magnitude with temperature change of a few hundred degrees. At low temperatures, viscous deformation is prohibited which leads to a buildup of elastic stresses during deformation, until the rock fails. This type of behavior characterizes brittle or elasto-plastic deformation. At high temperatures, on the other hand, the rock may accommodate high deformation rates through the viscous mechanism without excessive stress accumulation. This is a typical manifestation of the ductile flow regime.

The lithosphere is not only a region of large compositional heterogeneity, contrasting in this respect with asthenosphere, but it is also a locus of large temperature variations. In this context, it becomes necessary not only to resolve separate brittle and ductile flow regimes, but also to adequately model the brittle-ductile transition. Apart from many other complexities, this single

\footnotetext{
${ }^{*}$ Corresponding author. E-mail address: anton@gfz-potsdam.de (A.A. Popov)
} 
requirement makes lithospheric-scale modeling a challenging task.

This is further complicated by several factors, including: the presence of the Earth's free surface, where erosion and sedimentation processes take place; the occurrence of metamorphic reactions in rocks with changing temperature and pressure; the presence of spontaneously evolving largescale fault zones; by the inherent three-dimensional nature of most lithospheric-scale problems, and many others. All these complexities pose severe conceptual and implementation problems for lithospheric-scale modeling tools.

In recent decades, the geodynamic modeling community has accumulated significant experience in the development and application of numerical tools designed for modeling various geodynamic processes. These studies include Christensen and Harder (1991), Weinberg and Schmeling (1992), Bercovici (1993), Poliakov et al. (1993), Braun and Sambridge (1994), Bunge and Baumgardner (1995), Fullsack (1995), Trompert and Hansen (1996), Zhong and Gurnis (1996), Schmalholz et al. (2001), Babeyko et al. (2002), Tackley and Xie (2003), Moresi et al. (2003), Sobolev et al. (2005), Muhlhaus and Regenauer-Lieb (2005), Petrunin and Sobolev (2006), O'Neil et al. (2006), Gerya and Yuen (2007), Braun et al. (this issue), and Petrunin and Sobolev (this issue), which have all contributed to the development of geodynamic modeling techniques.

In our research we are mostly interested in highly dynamic deformation processes occurring at plate boundaries involving the combination of transform and compressional/extensional deformation. Such processes are essentially $3 \mathrm{D}$ and operate on a temporal scale of a few to several hundred million years and a spatial scale of hundreds to thousands of kilometers. Specific examples include continental collision processes in Tibet and transform deformation at the San Andreas Fault System and the Dead Sea Transform. Numerical techniques to handle such processes must be $3 \mathrm{D}$, should operate with an elasto-visco-plastic rheology, as this is the most adequate rheology to model lithospheric deformation, and must be suitable to model geological time-scale processes. Unfortunately, none of the tools available at present can be directly applied to these problems. The most suitable 3D codes either still do not include elastic deformation processes (Braun et al., this issue) or, being explicit codes (Petrunin and Sobolev, 2006, this issue), require very small steps for time integration and therefore are not efficient for modeling long-term deformation. The best candidate among existing tools is the $3 \mathrm{D}$ version of viscoelasto-plastic code I2ELVIS, which is currently under development (Gerya and Yuen, 2007). We note, however, that this code is based on a purely Eulerian approach and uses fully staggered finite-difference discretization. Staggered schemes are inherently stable (Shih et al., 1989), but the Eulerian approach substantially complicates treatment of the free surface.

With this in mind, we propose here a new code for lithospheric-scale modeling (SLIM3D). It is an implicit Arbitrary Lagrangian Eulerian Particle-in-Cell Finite Element code with a free surface, designed specifically for the thermo-mechanical modeling of deformation processes involving an elasto-visco-plastic lithospheric rheology on geologic time scales. SLIM3D is being designed to model deformation at plate boundaries (the focus of the recently formed geodynamic modeling group in GFZ-Potsdam). It is intended to complement the capability of the explicit code LAPEX3D already used in the same group for similar problems (Petrunin and Sobolev, 2006, this issue). SLIM3D is developed and implemented using the $\mathrm{C}++$ object-oriented programming language.

We emphasize that the potential advantage of SLIM3D over the other similar codes is in its three-dimensional nature combined with the adequate description of lithospheric deformation (rheology, strain localization, free surface etc.). To our knowledge, such a combination is unique 
at present. At the same time, we note that the code is still limited in certain aspects, which are already implemented in the other codes. These aspects are, for example, the multigrid solver (e.g. Moresi et al., 2003) and the adaptive mesh (e.g. Braun et al., this issue). Despite the present disadvantages, we suggest that SLIM3D has a good potential to be substantially improved in the future versions.

This paper is not intended to demonstrate the application of the code to certain geodynamic problems, but rather to introduce the method in general with several benchmarks and examples. The structure of the paper is as follows. In the next section, we describe the basic physical and rheological framework that we utilize for lithospheric scale modeling. In section 3 we outline implementation details of the code. In section 4, we test our tool with five benchmark problems which demonstrate aspects of elastic, viscous and plastic deformation mechanisms both separately and in combination. Finally, we briefly conclude our work and describe the direction we are planning to further develop our technique.

\section{Physical models}

\subsection{Conservation equations}

Lithospheric-scale deformation can be effectively characterized as a quasi-static thermomechanically coupled deformation process. Assuming a continuous media approximation, we can describe this process by the conservation equations of momentum:

$\frac{\partial \sigma_{i j}}{\partial x_{j}}+\rho g \hat{z}_{i}=0$,

and thermal energy:

$\frac{D U}{D t}=-\frac{\partial q_{i}}{\partial x_{i}}+r$

Here, $x_{i}(i=1,2,3)$ denote Cartesian coordinates, $\sigma_{i j}$ is the Cauchy stress tensor, $\rho$ is the material density, $g$ is the gravitational acceleration, $\hat{z}_{i}$ is the unit vector of the vertical axis pointing downward, $U$ is internal energy, $D / D t$ is the material time derivative, $q_{i}$ is the heat flux vector, $r$ is volumetric heat sources. In the above equations and in the rest of this paper, we use indicial notation and apply Einstein summation convention over repeated indices. Since there is no difference between covariant and contravariant components in the Cartesian coordinate system, all tensor indices are written as subscripts. For convenience we additionally explain notation, meaning, and dimension of basic quantities used in this paper in Table 1.

\subsection{Deviatoric-volumetric decomposition}

The thermo-rheological behavior of the rocks is more conveniently formulated in terms of the deviatoric-volumetric strain (stress) decomposition (e.g. Bonet and Wood, 1997). For the Cauchy stress tensor we may write:

$$
\tau_{i j}=\sigma_{i j}+p \delta_{i j}, \quad p=-\frac{1}{3} \sigma_{i i},
$$

where $\tau_{i j}$ is the Cauchy stress deviator and $p$ is hydrostatic pressure (positive in compression). The deviatoric strain rate tensor and the rate of volume change, respectively, may be written directly as: 
Table 1

Nomenclature

\begin{tabular}{|c|c|c|c|c|c|}
\hline \multicolumn{2}{|c|}{ Variable } & \multirow{2}{*}{$\begin{array}{l}\text { Meaning } \\
\text { Cartesian coordinates }\end{array}$} & \multicolumn{2}{|c|}{ Variable } & \multirow{2}{*}{$\begin{array}{l}\text { Meaning } \\
\text { Peierls stress }\end{array}$} \\
\hline$x_{i}$ & $\mathrm{~m}$ & & $\tau_{P}$ & $\mathrm{~Pa}$ & \\
\hline$t$ & $\mathrm{~s}$ & Time & $\varphi$ & $\left(^{\circ}\right)$ & Friction angle \\
\hline$v_{i}$ & $\mathrm{~m} \cdot \mathrm{s}^{-1}$ & Velocity vector & $c$ & $\mathrm{~Pa}$ & Cohesion \\
\hline$T$ & $\mathrm{~K}$ & Temperature & $\alpha$ & $\mathrm{K}^{-1}$ & Thermal expansivity \\
\hline$g$ & $\mathrm{~m} \cdot \mathrm{s}^{-2}$ & Gravitational acceleration & $C_{p}$ & $\mathrm{~J} \cdot \mathrm{kg}^{-1} \cdot \mathrm{K}^{-1}$ & Specific heat \\
\hline$\rho$ & $\mathrm{kg} \cdot \mathrm{m}^{-3}$ & Density & $\lambda$ & $\mathrm{W} \cdot \mathrm{m}^{-1} \cdot \mathrm{K}^{-1}$ & Thermal conductivity \\
\hline$K$ & $\mathrm{~Pa}$ & Bulk modulus & $A$ & $\mathrm{~W} \cdot \mathrm{kg}^{-1}$ & Radiogenic heat production \\
\hline$G$ & $\mathrm{~Pa}$ & Shear modulus & $q_{i}$ & $\mathrm{~W} \cdot \mathrm{m}^{-2}$ & Heat flux vector \\
\hline$B_{L}$ & $\mathrm{~Pa}^{-1} \cdot \mathrm{s}^{-1}$ & Diffusion creep constant & $U$ & $\mathrm{~J}$ & Internal energy \\
\hline$H_{L}$ & $\mathrm{~J} \cdot \mathrm{mol}^{-1}$ & Diffusion creep enthalpy & $p$ & $\mathrm{~Pa}$ & Pressure \\
\hline$B_{N}$ & $\mathrm{~Pa}^{-n} \cdot \mathrm{s}^{-1}$ & Dislocation creep constant & $\theta$ & - & Volumetric strain \\
\hline$H_{N}$ & $\mathrm{~J} \cdot \mathrm{mol}^{-1}$ & Dislocation creep enthalpy & $\tau_{i j}$ & $\mathrm{~Pa}$ & Deviatoric stress tensor \\
\hline$n$ & - & Dislocation creep exponent & $\dot{\varepsilon}_{i j}$ & $\mathrm{~s}^{-1}$ & Deviatoric strain rate tensol \\
\hline$B_{P}$ & $\mathrm{~s}^{-1}$ & Peierls creep constant & $\eta_{e f f}$ & $\mathrm{~Pa} \cdot \mathrm{s}$ & Effective viscosity \\
\hline
\end{tabular}

$\dot{\varepsilon}_{i j}=\frac{1}{2}\left(\partial v_{i} / \partial x_{j}+\partial v_{j} / \partial x_{i}\right)-\frac{1}{3}\left(\partial v_{k} / \partial x_{k}\right) \delta_{i j}, \quad \dot{\theta}=\partial v_{i} / \partial x_{i}$,

where $v_{i}$ is the spatial velocity vector. We adopt the second (Euclidean) norm as the effective scalar measure of deviatoric tensorial quantities. For an arbitrary tensor $a_{i j}$ the second norm is expressed as:

$a_{I I}=\left(a_{i j} a_{i j}\right)^{1 / 2}$.

\subsection{Continuity equation}

We include the effects of elastic compressibility and thermo-elasticity. In this case, the continuity equation can be conveniently coupled with the constitutive equation for hydrostatic pressure:

$$
\frac{D p}{D t}=-K\left(\dot{\theta}-\alpha \frac{D T}{D t}\right)
$$

Here, $K$ is the bulk modulus and $\alpha$ is the coefficient of thermal expansion. The presence of volumetric deformations evokes the following corrections of material density:

$\rho=\rho_{0}\left[1-\alpha\left(T-T_{0}\right)+\frac{p}{K}\right]$,

where $\rho_{0}$ is the density at reference temperature and zero pressure and $T_{0}$ is the reference temperature. Note that Eq. (7) can be easily replaced by the equation of state applicable for high pressure and temperature for models involving deep portions of the mantle.

\subsection{Additive decomposition and elasticity}




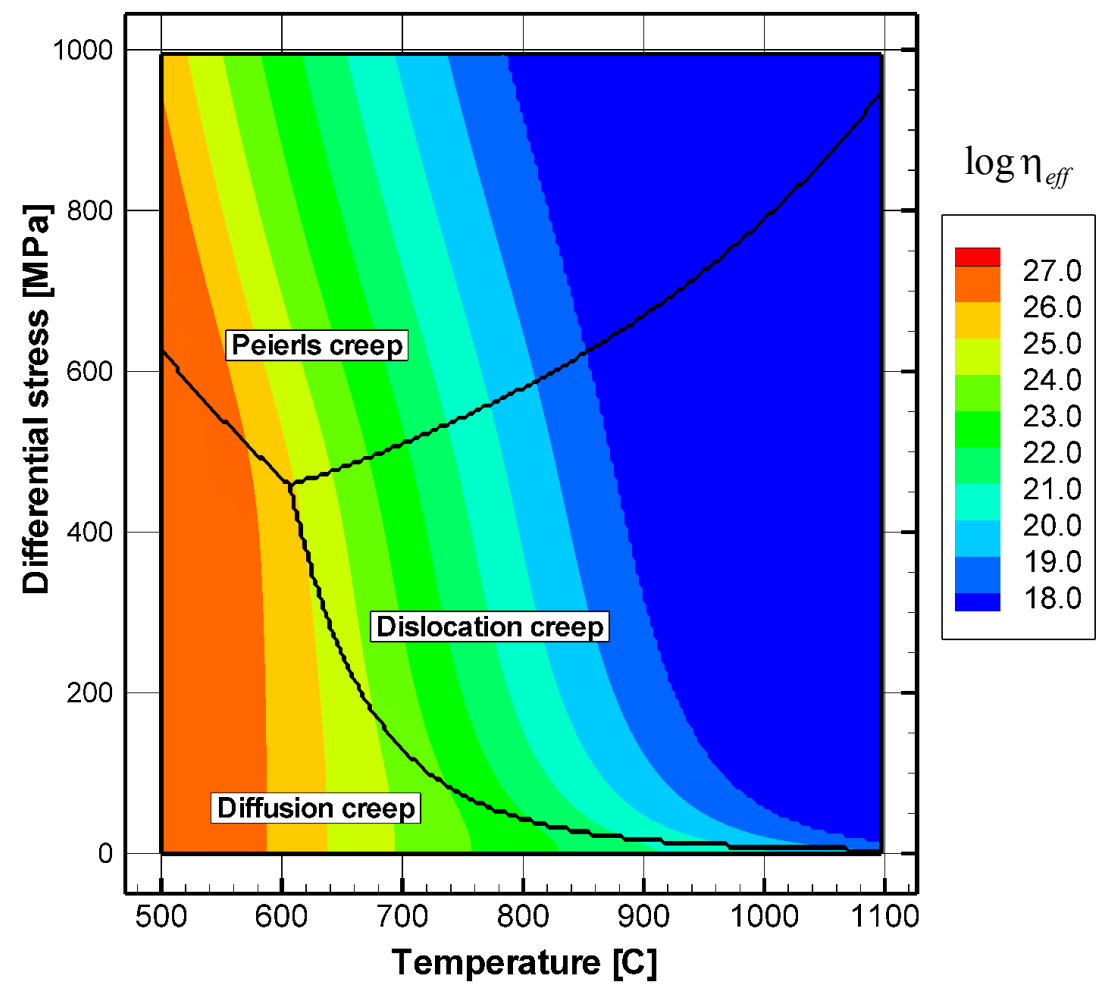

Figure 1 Logarithm of effective viscosity for dry olivine calculated using parameters from Kameyama et al. (1999). Temperature-stress domains in which each particular creep mechanism produces the largest strain rate are labeled. Black solid lines separate the domains. Viscosity is truncated to a reasonable range $10^{18}-10^{27}[\mathrm{~Pa} \cdot \mathrm{s}]$.

Taking benefit from the smallness of elastic strains, we adopt conventional additive decomposition (e.g. Simo and Hughes, 2000) of total deviatoric strain rate. The elastic, viscous and plastic components, respectively, can be written as follows:

$\dot{\varepsilon}_{i j}=\dot{\varepsilon}_{i j}^{e l}+\dot{\varepsilon}_{i j}^{v s}+\dot{\varepsilon}_{i j}^{p l}=\frac{1}{2 G} \hat{\tau}_{i j}+\frac{1}{2 \eta_{e f f}} \tau_{i j}+\dot{\gamma} \frac{\partial Q}{\partial \tau_{i j}}$,

where $G$ is the elastic shear modulus, $\hat{\tau}_{i j}$ is the objective stress rate (e.g. Bonet and Wood, 1997), $\eta_{\text {eff }}$ is the effective creep viscosity, $\dot{\gamma}$ is the plastic multiplier, and $Q$ is the plastic potential function (e.g. Simo \& Hughes, 2000). Note that for numerical integration of elastic stresses over the finite time step, we use the incrementally objective scheme of Hughes and Winget (1980). For more details see the next section of this paper.

\subsection{Ductile creep}

We adopt a detailed description of the ductile deformation component. The total viscous strain rate is additively decomposed into three temperature- and stress-dependent creep mechanisms, namely diffusion creep, dislocation creep and Peierls creep (see e.g. Kameyama et al., 1999). The corresponding effective creep viscosity is given by:

$$
\eta_{\text {eff }}=\frac{1}{2} \tau_{I I}\left(\dot{\varepsilon}_{L}+\dot{\varepsilon}_{N}+\dot{\varepsilon}_{P}\right)^{-1} \text {, }
$$

where $\tau_{I I}$ is effective differential stress (see Eq. 5), and $\dot{\varepsilon}_{L}, \dot{\varepsilon}_{N}$ and $\dot{\varepsilon}_{P}$ are the effective scalar strain rates due to the diffusion, dislocation and Peierls mechanisms, respectively. Specific expressions for each strain rate can be written as follows: 


$$
\begin{aligned}
& \dot{\varepsilon}_{L}=B_{L} \tau_{I I} \exp \left(-\frac{H_{L}}{R T}\right), \\
& \dot{\varepsilon}_{N}=B_{N}\left(\tau_{I I}\right)^{n} \exp \left(-\frac{H_{N}}{R T}\right), \\
& \dot{\varepsilon}_{P}=B_{P} \exp \left[-\frac{H_{P}}{R T}(1-\beta)^{2}\right]\left(\frac{\tau_{I I}}{\beta \tau_{P}}\right)^{s},
\end{aligned}
$$

where

$s=2 \beta(1-\beta) \frac{H_{P}}{R T}$.

In the above equations, $B_{L}, B_{N}, B_{P}$ and $H_{L}, H_{N}, H_{P}$ denote the creep parameter and activation enthalpy, respectively, of each correspondent mechanism, $R$ is the gas constant, $n$ is the power law exponent, $\tau_{P}$ is the Peierls stress, and $0<\beta<1$ is an adjustable approximation parameter. Note that in Eq. (12) we have adopted an asymptotic approximation of Peierls mechanism (see Kameyama et al. 1999), since the original equation is inappropriate for stresses below $O\left(10^{2}\right) \mathrm{MPa}$.

Here we assume that the creep parameter and activation enthalpy are constant for all creep mechanisms. In the general case, the creep parameter can depend on grain size (Karato et al., 2001), and activation enthalpy may be sensitive to pressure (Regenauer-Lieb and Yuen, 2004). If necessary, these complexities can be easily introduced in the presented numerical formulation.

In Fig. 1, we plot the effective logarithmic viscosity versus temperature and differential stress, assuming parameters for dry olivine from Kameyama et al. (1999). Each creep mechanism dominates over the others (produces a higher strain rate) in different temperature-stress domains. In Fig. 1, these domains are separated by black solid lines and labeled. Note that for olivine, most of effective viscosity reduction from extremely high $\left(10^{27} \mathrm{Pas}\right)$ to extremely low $\left(10^{18} \mathrm{Pas}\right)$ values occurs within a relatively narrow temperature range between 500 and $1000 \mathrm{C}$ for all stress levels.

\subsection{Brittle failure}

We describe brittle failure of rocks by the classical Mohr-Coulomb plasticity model (see e.g. Vermeer, 1990). The expression for the Mohr-Coulomb yield surface can be written as:

$F=\frac{1}{2}\left(\sigma_{\max }-\sigma_{\min }\right)+\frac{1}{2}\left(\sigma_{\max }+\sigma_{\min }\right) \sin \varphi-c \cos \varphi \leq 0$,

where $\sigma_{\max }$ and $\sigma_{\min }$ are the maximum and minimum principal stresses (negative in compression), $\varphi$ is the material angle of friction, $c$ is cohesion, $\frac{1}{2}\left(\sigma_{\max }-\sigma_{\min }\right)$ is the maximum differential stress, and $\frac{1}{2}\left(\sigma_{\max }+\sigma_{\min }\right)$ is the normal stress.

In the principal stress space, the Mohr-Coulomb yield surface can be represented as a hexagonal pyramid with a singular apex point in the tensile domain. Physically, this singularity means that the Mohr-Coulomb yield surface is inappropriate to model tensile failure of the rocks. A more adequate description in the context of lithospheric deformation would require coupling with the continuity equation to account for large plastic dilatation. Such sophisticated treatment of the tensile failure is not yet available. Therefore in this paper we adopt a standard ad-hoc approach and approximate the Mohr-Coulomb yield surface in the tensile domain with the Tresca criterion which is given by: 


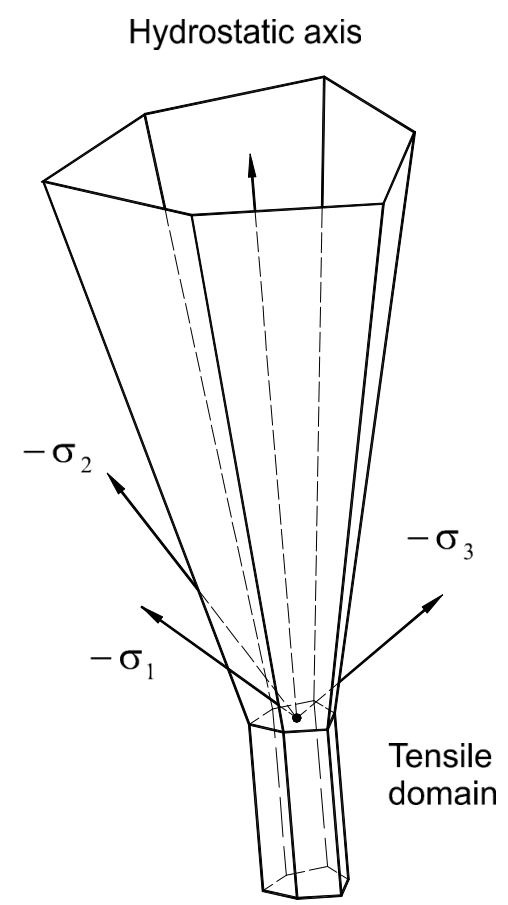

Figure 2 Schematic representation of the yield surface in principal stress space. In the tensile domain we use Tresca criterion.

$F=\frac{1}{2}\left(\sigma_{\max }-\sigma_{\min }\right)-c \cos \varphi \leq 0$.

The resulting composite yield surface is shown in Fig. 2.

Associative Mohr-Coulomb in 3D is essentially a multi-surface plasticity model which has socalled corner regions (Simo et al., 1988) in both yield surface and flow potential. Integration of such a model constitutes a particularly nontrivial task (Sloan and Booker, 1986; Larsson and Runesson, 1996; Borja et al., 2003). Another issue is related to the associativity of the flow rule, which significantly overestimates plastic dilatation of the rocks (e.g. Alejano and Alonso, 2005). We resolve both these issues by adopting the purely deviatoric corner-free Prandtl-Reuss flow rule (e.g. Zienkiewicz and Taylor, 2000), which is non-associative with the Mohr-Coulomb yield surface. In this case the plastic potential function takes the following simple form:

$Q=\tau_{I I}$.

The Prandtl-Reuss flow rule assumes complete plastic incompressibility (i.e. dilatation angle is zero), which is a suitable approximation for rocks in the large strain regime. At the same time, the integration of the plasticity model becomes considerably simpler due to a lack of corners in the plastic potential.

We approximate the degradation of the strength in faults and gouge zones by the strain softening model. The friction angle is assumed in the following product form:

$\varphi=\varphi_{0} D(\kappa)$,

where $\varphi_{0}$ is initial friction and $D(\kappa)$ is the function that controls degradation of the friction angle with progressive increase of the accumulated plastic strain. We assume $D(\kappa)$ in piecewise linear form. The accumulated plastic strain is given by:

$\kappa=\int_{t}\left(\dot{\varepsilon}_{i j}^{p l} \dot{\varepsilon}_{i j}^{p l}\right)^{1 / 2} d t$

We note that the adopted plasticity model with strain softening does not incorporate any length scale for the strain localization (e.g. Muhlhaus and Aifantis, 1991). Thus the theoretical thickness 
of the shear band is zero. In the numerical model the thickness of the shear band is limited from below by the element size. Therefore in the context of an adaptively refined grid one must incorporate the length-scale or certain regularization (e.g. Belytschko and Tabbara, 1993) to prevent the element size from reducing to very small values. In this paper we do not explicitly address this mesh-dependence issue, since at present we only consider uniform non-adaptive grids.

\subsection{Heat flow}

At present, we neglect latent heat effects due to phase change and assume internal energy in the form of linear function of temperature, i.e.:

$U=C_{p} T$,

where $C_{p}$ is specific heat.

We define the heat flux vector according to Fourier law as follows:

$q_{i}=-\lambda \delta_{i j} \frac{\partial T}{\partial x_{j}}$,

where $\lambda \delta_{i j}$ is the isotropic thermal conductivity tensor.

The volumetric heat sources include radiogenic heat and heat produced by visco-plastic deformation, i.e.:

$r=\rho A+\chi \tau_{i j}\left(\dot{\varepsilon}_{i j}^{v s}+\dot{\varepsilon}_{i j}^{p l}\right)$,

where $A$ is radiogenic heat per unit mass and $0 \leq \chi \leq 1$ is the constant regulating degree of thermo-mechanical feedback. This coupling constant is an ad-hoc approach that effectively accounts for the processes which we do not include in our models, such as heat transport by the fluids, etc.

\subsection{Boundary conditions}

The conservation equations (1) and (2) must be complemented with initial and boundary conditions. This may include specified velocity, temperature, stress and heat flux. Additionaly, in the context of lithospheric-scale modeling it becomes necessary to approximate the boundary conditions at the base of the lithosphere. In this paper we use the classical Winkler foundation (see e.g. Fig. 5 in Regenauer-Lieb, 2006), which assumes zero viscous drag forces and takes into account buoyancy forces. The boundary stress tensor of the Winkler foundation can be parameterized as follows:

$\bar{\sigma}_{i j}=-\left[p_{0}+\rho_{\text {ext }} g\left(z-z_{0}\right)\right] \delta_{i j}$,

where $p_{0}$ is pressure at the reference surface, $\rho_{\text {ext }}$ is the density of the external material, and $z$ and $z_{0}$ are the vertical coordinates of the bottom boundary and the reference surface, respectively. Typically, we use the initial position of the bottom boundary as the reference surface. The pressure at the reference surface is assigned as total weight divided by the depth of the model. Other non-standard boundary conditions include more complicated effects such as erosion on the free surface and material in-flux and out-flux. These effects are considered in more detail in the next section. 


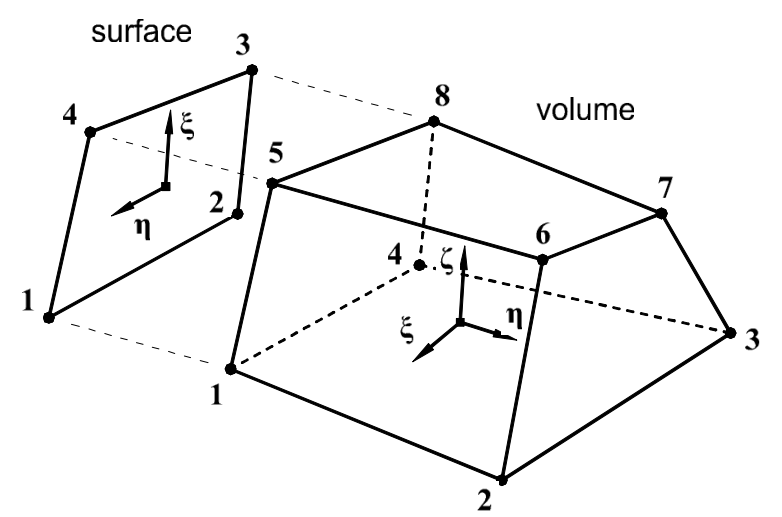

Figure 3 Finite element discretization of volume (hexahedrons) and surface (quadrilaterals). Shown are the local coordinate systems and local numbers of the nodes.

\section{Numerical algorithms}

\subsection{Spatial discretization}

We build numerical models of lithospheric-scale deformation on the basis of the Finite Element Method (e.g. Hughes, 1987; Zienkiewicz and Taylor, 2000; Belytschko et al., 2000). Following the Galerkin procedure (e.g. Belytschko et al., 2000), we transform the momentum balance equation (1) into a corresponding nodal force residual:

$$
f_{i I}=\int_{\Omega} \frac{\partial N_{I}}{\partial x_{j}} \sigma_{i j} d \Omega-\int_{\Omega} N_{I} \rho g \hat{z}_{i} d \Omega-\int_{\Gamma} N_{I} \bar{\sigma}_{i j} n_{j} d \Gamma=0 .
$$

Similarly, we approximate the energy balance equation (2) by a nodal power residual:

$$
w_{I}=\int_{\Omega} N_{I} \rho \frac{D U}{D t} d \Omega-\int_{\Omega} \frac{\partial N_{I}}{\partial x_{i}} q_{i} d \Omega-\int_{\Omega} N_{I} r d \Omega+\int_{\Gamma} N_{I} \bar{q}_{i} n_{i} d \Gamma=0 .
$$

Here $N$ denotes the nodal shape function (Zienkiewicz and Taylor, 2000), $I$ is the nodal index, $\bar{\sigma}_{i j}$ and $\bar{q}_{i}$ are surface stress and surface heat flux, respectively, $n_{i}$ is the outward unit normal, and $\Omega$ and $\Gamma$ stand for the volume and surface of the domain.

We employ hexahedral finite elements with linear interpolation functions (e.g. Zienkiewicz and Taylor, 2000) to approximate volume integrals in the above equations, and similar quadrilateral elements to approximate surface integrals (see Fig. 3). Element integrals are expressed in terms of parametric coordinates and evaluated over the unit cube or square using numerical integration (e.g. Belytschko et al., 2000). This stage is directly followed by assembly of the global residual equations (23) and (24) from element contributions (e.g. Hughes, 1987).

In each element, the shape function derivatives with respect to global coordinates are computed using standard coordinate transformation (summation over repeated nodal indices is implied):

$$
\frac{\partial N_{I}}{\partial x_{i}}=J_{i j}^{-1} \frac{\partial N_{I}}{\partial \xi_{j}}, \quad J_{i j}=\frac{\partial N_{I}}{\partial \xi_{i}} x_{j I},
$$

where $\xi_{i}$ are the local coordinates and $J_{i j}$ denotes Jacobian matrix. The unit outward normal vector is evaluated according to:

$n_{i}=J^{-1} \hat{n}_{i}, \quad J=\left(\hat{n}_{i} \hat{n}_{i}\right)^{1 / 2}$,

where $J$ stands for surface Jacobian and $\hat{n}_{i}$ is the cross-product between two surface tangent 
vectors, which can be expressed as follows:

$\hat{n}_{i}=\varepsilon_{i j k} t_{j}^{(1)} t_{k}^{(2)}, \quad t_{i}^{(a)}=\frac{\partial N_{I}}{\partial \xi_{a}} x_{i I}, \quad a=1,2$.

Here $t_{i}^{(a)}$ are the surface tangent vectors and $\varepsilon_{i j k}$ is the Levi-Civita permutation symbol. To ensure a positive determinant of the Jacobian matrix and outward orientation of the unit normal vector, we perform appropriate control over the numbering of nodes in the element.

\subsection{Locking and Hourglass}

In nearly incompressible problems the finite element mesh is prone to locking, which manifests itself in severe underestimation of velocities/displacements (e.g. Belytschko et al., 2000). Locking is cured either by suitable under-integration of stress terms (Malkus and Hughes, 1978) or by using higher interpolation order for displacements (velocities) than for the stresses (e.g. Zienkiewicz and Taylor, 2000). Both approaches are essentially equivalent.

We suppress locking by evaluating constitutive equations only in one point per element (Flanagan and Belytschko, 1981). This approach implies that strains (strain rates) and other spatial gradients are evaluated using the following element-average derivatives of the shape functions:

$b_{i I}=\frac{1}{V_{E}} \int_{\Omega} \frac{\partial N_{I}}{\partial x_{i}} d \Omega$.

where $V_{E}$ is the element volume. For element integrals, we use standard quadrature.

The hexahedral elements employed here violate the so-called LBB stability condition named after Ladyzhenskaya (1969), Babuska (1973) and Brezzi (1974), which leads to mesh artifacts known as "hourglass modes" (e.g. Flanagan and Belytschko, 1981) (see also Fig. 4a). Despite this, we use them together with careful monitoring of the stress and displacements fields, because the more stable quadratic element is computationally very expensive in $3 \mathrm{D}$ problems. With the same element resolution, quadratic interpolation requires roughly an order of magnitude more nodes (degrees of freedom) than the linear interpolation.

We have tested some of the anti-hourglass techniques available for hexahedral (quadrilateral) and tetrahedral (triangular) elements (see e.g. Flanagan and Belytschko, 1981; Liu et al., 1998; Bonet et al., 2001; Reese, 2003; Puso and Solberg, 2006). In certain problems, such as the Rayleigh-Taylor instability with large abrupt viscosity variations (see Fig. 4), none of stabilization technique we tested was able to prevent the hourglass modes. We have inferred that the critical factor is the relatively high confining pressure compared to deviatoric stress in the low viscosity domain.

The magnitude of the confining pressure can be reduced by replacing the material density with the differential density $\Delta \rho=\rho-\rho_{\text {ref }}$. Here $\rho_{\text {ref }}$ denotes the arbitrary chosen constant reference density. With the differential density approach, the numerical solution produces the dynamic pressure $\Delta p$. To evaluate the constitutive equations properly, it becomes necessary to augment the dynamic pressure with a lithostatic component $\rho_{\text {ref }} g\left(z-z_{\text {free }}\right)$. Here $z$ and $z_{\text {free }}$ denote the vertical coordinates of the integration point and the free surface, respectively. The density of the 
$[A]$ total density formulation

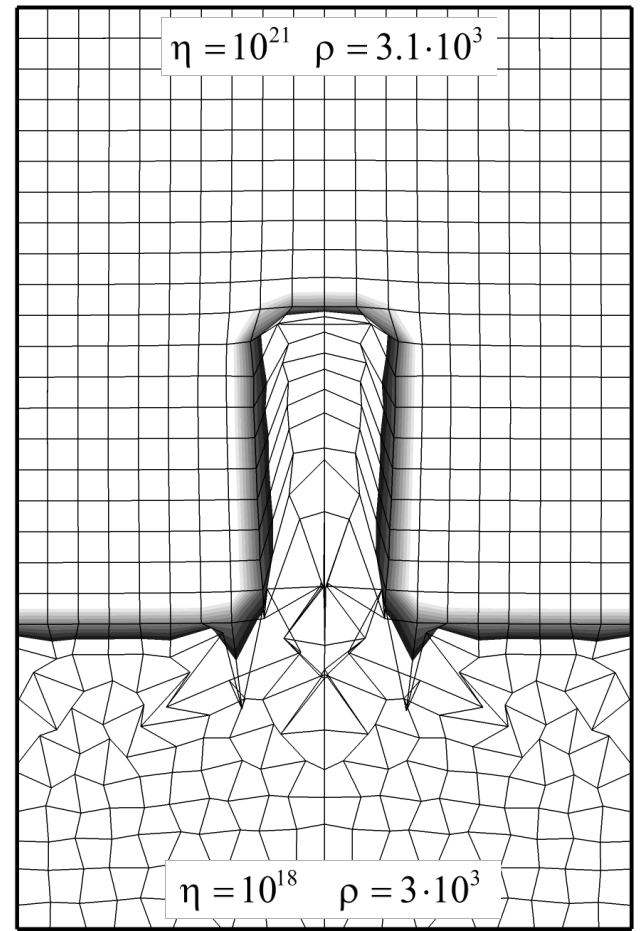

[B] differential density formulation

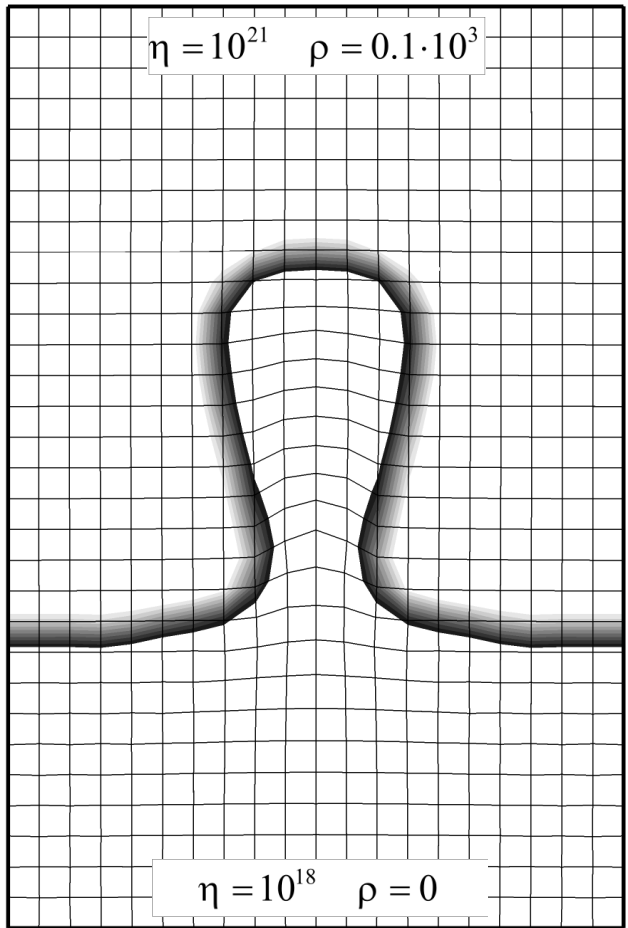

Figure 4 Grid distortion for a single time step of the Rayleigh-Taylor instability problem with large (3 orders of magnitude) abrupt viscosity variation. (a) total density formulation, (b) differential density formulation. Gradient curve indicates boundary between the layers. Shown are the viscosity and density of upper and lower layers.

external material must also be set to the differential value $\Delta \rho_{\text {ext }}=\rho_{\text {ext }}-\rho_{\text {ref }}$. Similarly, the Winkler condition must be applied at the Lagrangian free surface with the differential density equal to negative reference density i.e. $\Delta \rho_{\text {air }}=-\rho_{\text {ref }}$.

According to our experience, this simple rearrangement of the computational scheme is sufficient to remove the hourglass modes even in the Rayleigh-Taylor instability problem mentioned above. Figs. $4 \mathrm{a}$ and $4 \mathrm{~b}$ show the mesh distortion during a typical time step for the total and differential density formulations, respectively. The total density formulation exhibits uncontrollable oscillation while differential density formulation behaves stably.

We note, however, that despite the fact that the differential density technique can extend stability margins in certain problems, there is no guarantee that it will work in other problems. Therefore, we suggest that this technique should be used only in conjunction with careful monitoring of the stress and displacements fields. Additionally, one can use spatial averaging over the neighboring nodes (e.g. Fullsack, 1995; Braun et al., this issue) to enhance the stability.

As an alternative to finite elements with bilinear interpolation for velocities and constant pressure, fully staggered finite difference discretization can be used (Harlow and Welsh, 1965), which appear to be non-oscillatory (for discussion, see Shih et al., 1989). Successful application of this discretization in geodynamic problems is presented in Gerya and Yuen (2007). However this approach, despite stability, is restricted to pure Eulerian formulations. In lithospheric scale modeling, we consider this restriction as unfavorable because it complicates tracking of the free surface. 


\subsection{Time discretization and primary variables}

We use the backward Euler method as the primary time discretization algorithm for both the momentum and energy balance equations. This approach is referred to as implicit and first order accurate. The first order accuracy brings advantageous stability, which is missed by the second order accurate trapezoidal rule (Ortiz and Popov, 1985). In the adopted context, integration of all quantities becomes particularly simple, e.g. for velocities we can write:

$\Delta u_{i}=v_{i} \Delta t$

where $\Delta u_{i}$ is incremental displacement vector and $\Delta t={ }^{n+1} t-{ }^{n} t$ is the time step. Here the left superscript indicates the time step index. In the remainder of this paper we omit the index of the current time step for notational clarity, e.g. we simply write: $\Delta t=t-{ }^{n} t$.

The simplicity of the integration scheme allows us to choose either velocities or incremental displacements as primary kinematical variables, irrespective of the employed rheology or kinematical formulation. Indeed, it is clear from the integration formula (29) that both approaches are essentially equivalent. For convenience we prefer incremental displacements. Note that all results presented below are readily extendable to the velocity formulation merely by simple scaling.

We do not separate pressure as an independent variable, since we use a compressible formulation. However, in typical problems the near-incompressible behavior is recovered in the asthenosphere domain. We treat this additional difficulty during the linear solution stage by appropriate damping of the stiffness parameters (see section 3.8 for more details). For the heat balance equation we use temperature as the primary variable.

\subsection{Kinematical formulation and solution scheme}

We employ the Arbitrary Lagrangian-Eulerian kinematical formulation (Hirt et al., 1974) to account for material advection. A typical calculation step is subdivided into three major stages:

(i) In the first stage, we solve the discretized residual equations (23) and (24) by the Newton Raphson iterative method (e.g. Belytschko et al., 2000). During iterations we conveniently treat advection terms implicitly by means of the Updated Lagrangian formulation (Bathe et al., 1975). The advantage is that Lagrangian treatment does not limit the time step by the amount of rigid body motion, in contrast with the Eulerian approach. In the Lagrangian context, only the amount of material straining poses an actual kinematic limit for the time step.

(ii) In the second stage, we perform mesh adaptation (regridding) such that the new mesh fits the free surface, the moving (stretching) calculation window and simple material interfaces without overturns or self-intersections. The flexibility of the approach is that regridding can be done either in each step or after a certain number of steps, which is favorable in the case that advection is not the dominant type of nonlinearity in the system (e.g. the onset of plastic localization). Regridding is also the easiest way to implement boundary material fluxes (erosion, sedimentation, etc.).

(iii) In the third and final stage, we perform consistent remapping of all solution variables onto the updated mesh. Our algorithm is based on the particle-in-cell approach which was initially developed by Harlow and Welsh (1965) and subsequently has been widely used in geodynamic applications (Moresi et al., 2003; Tackley and Xie, 2003; O’Neil et al., 2006; 
Gerya and Yuen, 2007).

We explain each calculation stage in more detail in the following sections. The overall computational flowchart is summarized in Table 2 .

\subsection{Hughes-Winget scheme}

A constitutive model of advection dominated flow that includes memory effects (e.g. Fullsack, 1995) should be objective, i.e. it should ensure conservation of tensor quantities (e.g. Muhlhaus and Regenauer-Lieb, 2005). This can be formally achieved by using time-continuous (infinitesimal) objective stress rates (e.g. Bonet and Wood, 1997). However, since our practical interest is implicit large-step integration of the flow, we use the Hughes-Winget scheme (Hughes-Winget 1980) to ensure objectivity in an incremental, rather than an infinitesimal, sense.

The central point of the scheme is the following second order accurate approximation for an incremental displacement gradient:

$h_{i j}=\frac{\partial \Delta u_{i}}{\partial^{n+1 / 2} x_{j}}, \quad{ }^{n+1 / 2} x_{j}={ }^{n} x_{j}+\frac{1}{2} \Delta u_{j}$,

where ${ }^{n+1 / 2} x_{j}$ is the mid-point material configuration. In each element, we compute $h_{i j}$ using the mid-point element-average derivatives of shape functions (see Eq. 28) as follows:

$h_{i j}=\Delta u_{i I}{ }^{n+1 / 2} b_{j I}$.

We note that the mid-point scheme in Eq. (30) effectively eliminates the influence of rigid body motion on the strain components (Hughes and Winget, 1980). This property contrasts with pure Eulerian formulation, which is restricted to differentiation only with respect to the current configuration.

Deviatoric and volumetric strain increments to be used in constitutive equations are computed, respectively, as follows:

$\varepsilon_{i j}=\frac{1}{2}\left(h_{i j}+h_{j i}\right)-\frac{1}{3} h_{k k} \delta_{i j}, \quad \theta=h_{i i}$.

Correspondent rate quantities are approximated by:

$\dot{\varepsilon}_{i j}=\frac{\varepsilon_{i j}}{\Delta t}, \quad \dot{\theta}=\frac{\theta}{\Delta t}$.

According to the Hughes-Winget scheme, rotation of stress from the previous time step is governed by the following orthogonal tensor (for more details see Hughes and Winget, 1980):

$R_{i j}=\left(\delta_{i k}-\frac{1}{2} \omega_{i k}\right)^{-1}\left(\delta_{k j}+\frac{1}{2} \omega_{k j}\right), \quad \omega_{i j}=\frac{1}{2}\left(h_{i j}-h_{j i}\right)$.

The approximations (30) - (34), despite their simplicity, produce stable and accurate results even for large strain and rotation increments (e.g. Rashid, 1993).

\subsection{Incremental constitutive equations}

Incremental expansion of the continuity equation (6) gives the following updated pressure, if the reactional (phase transformation) volume changes are neglected:

$p={ }^{n} p-K \theta+K \alpha\left(T-{ }^{n} T\right)$.

To update the deviatoric stress, we use a two-step predictor-corrector procedure (e.g. Simo and Hughes, 2000). In the first step, the plastic multiplier in equation (8) is assumed to be zero and 


\section{Table 2}

Overall computational flowchart

\section{Newton-Raphson solution}

1. Update stresses \& heat fluxes in the elements

2. Assemble \& solve linear systems

$$
\begin{aligned}
& \boldsymbol{\delta} \mathbf{u}=-\mathbf{K}_{(k)}^{-1} \mathbf{f}_{(k)}, \quad \boldsymbol{\delta} \mathbf{T}=-\mathbf{E}_{(k)}^{-1} \mathbf{w}_{(k)} \\
& \Delta \mathbf{u}_{(k+1)}=\Delta \mathbf{u}_{(k)}+\boldsymbol{\delta} \mathbf{u}, \quad \mathbf{T}_{(k+1)}=\mathbf{T}_{(k)}+\boldsymbol{\delta} \mathbf{T}
\end{aligned}
$$

3. Update displacement and temperature

4. Update coordinates

5. Check convergence

6. go to step (1) if necessary

\section{Regridding and Remapping}

1. Save history increment to markers

2. Advect markers by mesh

3. Adapt mesh to fit the free surface

4. Apply erosion boundary condition

5. Map markers onto adapted mesh

6. Count number of markers per cell

7. Insert/delete markers where necessary

8. Interpolate properties and history to mesh

the correspondent trial visco-elastic stress is evaluated. In the second step, the Mohr-Coulomb yield surface is checked (Eq.14), and if violated, the trial stress is modified to remain on the yield surface. This gives rise to a nonzero plastic multiplier and corresponding plastic strain increment.

Combining the Hughes-Winget scheme with the analytical integration of equation (8) over the time step we obtain the following expression for trial deviatoric stress:

$\tau_{i j}^{t r}=2 \eta_{C R} \dot{\varepsilon}_{i j}+\alpha_{C R} R_{i k}^{n} \tau_{k l} R_{j l}$,

where ${ }^{n} \tau_{k l}$ is the Cauchy stress deviator from previous time step, $R_{i j}$ is the incremental rotation tensor (see Eq. 34), and $\eta_{C R}$ and $\alpha_{C R}$ are the effective viscosity and relaxation ratio of viscoelastic creep, respectively, given by:

$\eta_{C R}=\eta_{e f f}\left(1-\alpha_{C R}\right), \quad \alpha_{C R}=\exp \left(-\frac{\Delta t}{t_{M}}\right), \quad t_{M}=\frac{\eta_{e f f}}{G}$.

Here $t_{M}$ stands for Maxwell time.

Equation (36) shows that the magnitude of Maxwell time does not pose the upper limit for the time step (see also Bailey, 2006). Indeed, in typical calculations we choose time steps equal to tens of thousands years. In the asthenosphere, where temperature is high enough to sufficiently decrease the viscosity, the following purely viscous limit is recovered:

$\frac{\Delta t}{t_{M}}>>1, \quad \lim _{\alpha_{C R} \rightarrow 0} \tau_{i j}^{t r}=2 \eta_{e f f} \dot{\varepsilon}_{i j}$.

In the upper crustal domain, on the other hand, the relatively low temperatures make the viscosity so large that Equation (36) degenerates into the corresponding elastic end-member: 
$\frac{\Delta t}{t_{M}}<<1, \quad \lim _{\alpha_{C R} \rightarrow 1} \tau_{i j}^{t r}=2 G \varepsilon_{i j}+R_{i k}^{n} \tau_{k l} R_{j l}$.

However, due to the presence of the Mohr-Coulomb stress limiter, most of deformation in the upper crustal domain manifests itself in the form of plastic localization.

Apart from temperature dependence, the effective viscosity depends also on stress, and this introduces nonlinearity into the equation (36). This issue can be resolved, in principle, with good stress estimation from the previous time step. However, we have concluded from our experiments that this explicit approach severely restricts stable calculation steps due to stress oscillations. The alternative is, therefore, implicit treatment of nonlinearity by solving the nonlinear equation (36) for a trial deviatoric stress.

The simplest fixed point iteration cannot be used to this end, since in our case it may produce infinite loops without convergence. Hence, we have adopted the simple and reliable root-solving algorithm FZERO (Shampine and Watts, 1970), which is based on a combination of the bisection and secant methods.

Prior to solution we rewrite the tensorial equation (36) in scalar residual form by taking the difference between norms of its left- and right hand sides. The solution is achieved when the residual is reduced to a sufficiently small relative tolerance. Typically, very few iterations (around 5-7) are required by FZERO to converge.

For numerical stability reasons, we remove stress dependence in the effective viscosity at differential stress levels higher than $O\left(10^{3}\right) \mathrm{MPa}$, which is far higher than expected in typical problems. Finally, in the numerical simulations we truncate effective viscosity to the reasonable range $10^{18}-10^{27}$ Pas.

After the visco-elastic stress predictor step, we check the yield surface to determine whether a plastic stress correction is required. In the following, we introduce basic elements of the procedure, whereas more details are outlined in Appendix A.

Plastic stress correction starts with spectral decomposition of the trial deviatoric stress tensor by the optimized 3D Jacobi eigenvalue algorithm (Press et al., 2002):

$\tau_{i j}^{t r}=\sum_{A=1}^{3} \tau_{A}^{t r} m_{i j}^{(A)}$.

Here $\tau_{A}^{t r}$ denotes the trial principal deviatoric stresses, $m_{i j}^{(A)}$ are the correspondent spectral directions.

In the next stage, we evaluate the trial yield surface (see Appendix A):

$F^{t r}=\frac{1}{2}\left(\tau_{\max }^{t r}-\tau_{\min }^{t r}\right)+\frac{1}{2}\left(\tau_{\max }^{t r}+\tau_{\min }^{t r}\right) \sin \varphi-p \sin \varphi-c \cos \varphi$.

For simplicity, the friction angle in the above equation is computed using the magnitude of accumulated plastic stain from previous time step, i.e. $\varphi=\varphi_{0} D\left({ }^{n} \kappa\right)$.

As soon as the trial deviatoric stress departs from the yield surface ( $F^{t r}>0$ condition is met), we undertake the following correction:

$\tau_{i j}=\alpha_{P L} \tau_{i j}^{t r}$,

where $0<\alpha_{P L}<1$ is the plastic scaling ratio, which is given by: 


$$
\alpha_{P L}=\frac{2(p \sin \varphi+c \cos \varphi)}{(1+\sin \varphi) \tau_{\max }^{t r}-(1-\sin \varphi) \tau_{\min }^{t r}} .
$$

For simplicity, we assume that both the effective viscosity of visco-elastic creep and the trial stress remain unaltered during plastic stress correction.

The magnitude of plastic strain rate is readily computed as follows:

$\dot{\gamma}=\frac{1}{2 \eta_{C R}}\left(1-\alpha_{P L}\right) \tau_{I I}^{t r}$.

Integrating the above expression by the Backward Euler algorithm we obtain the following relation for the plastic strain increment:

$\Delta \kappa=\frac{\Delta t}{2 \eta_{C R}}\left(1-\alpha_{P L}\right) \tau_{I I}^{t r}$,

which produces asymptotically exact results for both viscous and elastic cases. Corresponding limit expressions are given by:

$\lim _{\alpha_{C R} \rightarrow 0} \frac{\Delta t}{2 \eta_{C R}}=\frac{\Delta t}{2 \eta_{\text {eff }}}, \quad \lim _{\alpha_{C R} \rightarrow 1} \frac{\Delta t}{2 \eta_{C R}}=\frac{1}{2 G}$.

Accumulated plastic strain for the next time step is updated by:

$\kappa={ }^{n} \kappa+\Delta \kappa$.

For the Updated Lagrangian kinematical formulation, the material and partial time derivatives are coincident. We can therefore approximate the rate of internal energy in each integration point by the following first order difference:

$$
\frac{D U}{D t}=C_{p} \frac{T_{I} N_{I}-{ }^{n} T_{I} N_{I}}{\Delta t} \text {. }
$$

For consistency with the Hughes-Winget scheme, we evaluate the spatial temperature gradient with respect to the mid-point material configuration (see Eq. 30). Thus, the finite element approximation for the heat flux vector can be written as (see Eq. 28):

$q_{i}=-\lambda T_{I}^{n+1 / 2} b_{i I}$.

Heat source due to dissipation of mechanical energy can be re-expressed as:

$\tau_{i j}\left(\dot{\varepsilon}_{i j}^{v s}+\dot{\varepsilon}_{i j}^{p l}\right)=\tau_{I I}\left(\frac{\tau_{I I}}{2 \eta_{e f f}}+\dot{\gamma}\right)$.

The basic steps of the stress update procedure are summarized in Table 3 . We note that this sequence is repeated every iteration of every time step in each integration point.

\subsection{Nonlinear solver}

At each time step, we solve the coupled system of discretized residual equations (23) and (24) by the full Newton-Raphson iterative method (e.g. Belytschko et al., 2000). We neglect coupling terms in the Jacobian matrix. Therefore, at each iteration we solve the following two systems of linear equations:

$$
\begin{aligned}
& \boldsymbol{\delta} \mathbf{u}=-\mathbf{K}_{(k)}^{-1} \mathbf{f}_{(k)}, \quad \Delta \mathbf{u}_{(k+1)}=\Delta \mathbf{u}_{(k)}+\boldsymbol{\delta} \mathbf{u}, \\
& \boldsymbol{\delta} \mathbf{T}=-\mathbf{E}_{(k)}^{-1} \mathbf{w}_{(k)}, \quad \mathbf{T}_{(k+1)}=\mathbf{T}_{(k)}+\boldsymbol{\delta} \mathbf{T} .
\end{aligned}
$$

Here, the right subscript $(k)$ denotes iteration index, $\mathbf{f}$ is the out-of-balance nodal force vector 


\section{Table 3}

Stress update in the integration point

1. Compute displacement gradient

$$
\begin{aligned}
& h_{i j}=\Delta u_{i I}{ }^{n+1 / 2} b_{j I} \\
& \varepsilon_{i j}=\frac{1}{2}\left(h_{i j}+h_{j i}\right)-\frac{1}{3} h_{k k} \delta_{i j}, \quad \theta=h_{i i} \\
& p={ }^{n} p-K \theta+K \alpha\left(T-{ }^{n} T\right) \\
& \tau_{i j}^{t r}=2 \eta_{C R} \dot{\varepsilon}_{i j}+\alpha_{C R} R_{i k}{ }^{n} \tau_{k l} R_{j l} \\
& \tau_{i j}^{t r}=\sum_{A=1}^{3} \tau_{A}^{t r} m_{i j}^{(A)} \\
& F^{t r}<0 \Rightarrow \quad \operatorname{Exit} \\
& \alpha_{P L}=\frac{2(p \sin \varphi+c \cos \varphi)}{(1+\sin \varphi) \tau_{\max }^{t r}-(1-\sin \varphi) \tau_{\min }^{t r}} \\
& \tau_{i j}=\alpha_{P L} \tau_{i j}^{t r} \\
& \kappa={ }^{n} \kappa+\frac{\Delta t}{2 \eta_{C R}}\left(1-\alpha_{P L}\right) \tau_{I I}^{t r}
\end{aligned}
$$

8. Update stress

9. Update plastic strain

(Eq. 23), $\mathbf{w}$ is the out-of-balance nodal power vector (Eq. 24), $\boldsymbol{\Delta} \mathbf{u}$ is the incremental displacement (total velocity) vector, $\mathbf{T}$ is the nodal temperature vector, $\mathbf{K}=\partial \mathbf{f} / \Delta \mathbf{u}$ is the mechanical tangent matrix, $\mathbf{E}=\partial \mathbf{w} / \partial \mathbf{T}$ is the thermal tangent matrix, and $\delta \mathbf{u}$ and $\boldsymbol{\delta} \mathbf{T}$ are iterative correction vectors for incremental displacement and temperature, respectively.

At each iteration, we update nodal coordinates similarly to incremental displacements $\mathbf{x}_{(k+1)}=\mathbf{x}_{(k)}+\boldsymbol{\delta} \mathbf{u}$, while all the element integrals are evaluated over the latest updated coordinates (Updated Lagrangian formulation).

We achieve a coupled thermo-mechanical solution by:

(i) Calculating effective viscosity and density using the latest updated temperature,

(ii) Calculating mechanical dissipation using the latest available stress and strain rate,

(iii) Simultaneously solving the force balance and energy balance equations.

In the context of the Newton-Raphson method, it is necessary to carefully select the initial guess, since it can significantly reduce computation time. At the first time step we take zero displacements and an initial temperature distribution. For each subsequent time step we use the previous converged solution.

Constrained degrees of freedom are removed from the tangent matrices. To facilitate convergence only at the first iteration of the first time step, we add corrections related to removed degrees of freedom to the right hand side vectors.

We employ the so-called line search procedure (see e.g. Press et al., 2002; Crisfield, 1983) to stabilize iterative solution of the mechanical equation. The displacement update formula with line search is modified to the following form:

$\Delta \mathbf{u}_{(k+1)}=\Delta \mathbf{u}_{(k)}+\alpha \boldsymbol{\delta} \mathbf{u}$.

Here $0<\alpha<1$ is the damping parameter, which is chosen to satisfy the following condition: 


$$
\left\|\mathbf{f}_{(k+1)}\right\|<\left\|\mathbf{f}_{(k)}\right\| \text {. }
$$

We bisect the time step and restart the overall equilibrium iteration in case the above criterion cannot be fulfilled. This technique forms the core of our adaptive time-stepping algorithm. Initially we specify the uniform time step; the algorithm then performs bisections whenever convergence problems are experienced. When iteration becomes stable again, the algorithm tries to increase the time step. However it keeps in memory the last converged solution, to restore stable iteration in the case of unsuccessful time step increases. To further enhance convergence in the strain localization problems, more sophisticated time-stepping methods may be used such as the method of subplane control functions (Geers, 1999).

We terminate the Newton-Raphson iteration as soon as the following criteria are satisfied:

$$
\|\boldsymbol{\delta} \mathbf{u}\|<\varepsilon\left\|\Delta \mathbf{u}_{(k+1)}\right\| \text { and }\|\boldsymbol{\delta} \mathbf{T}\|<\varepsilon\left\|\mathbf{T}_{(k+1)}\right\| \text {, }
$$

where $\varepsilon$ is a small tolerance, typically $\varepsilon=10^{-3}$.

\subsection{Linearization and linear solver}

We assume the following simplified approximations for the tangent matrices (Appendix B):

$$
\begin{aligned}
& K_{i k J J}=\int_{\Omega} \frac{\partial N_{I}}{\partial x_{j}} C_{i j k l} \frac{\partial N_{J}}{\partial x_{l}} d \Omega+\int_{\Gamma} N_{I}\left(\rho_{\text {ext }} g n_{z} \hat{z}_{i} \hat{z}_{k}\right) N_{J} d \Gamma, \\
& E_{I J}=\int_{\Omega} N_{I} \frac{\rho C_{p}}{\Delta t} N_{J} d \Omega+\int_{\Omega} \frac{\partial N_{I}}{\partial x_{i}} \lambda \frac{\partial N_{J}}{\partial x_{i}} d \Omega .
\end{aligned}
$$

Here, $C_{i j k l}$ is the material tangent operator (Simo and Taylor, 1985), $n_{z}$ is the vertical component of outward normal vector, and $\hat{z}_{i}$ are the components of the unit vector of the vertical axis (downward positive). The first term in Eq. (56) corresponds to linearization of the internal stress term, while the second term is caused by the Winkler boundary condition.

Depending on type of the flow (visco-elastic or elasto-visco-plastic), the material tangent operator takes the following simple form:

$$
C_{i j k l}=K \delta_{i j} \delta_{k l}+\left\{\begin{array}{ll}
2 G_{C R} I_{i j k l}^{D} & \text { if } F^{t r} \leq 0 \\
2 G_{P L} I_{i j k l}^{D}+\tau_{i j}^{t r} \widehat{g}_{k l} & \text { if } F^{t r}>0
\end{array}\right. \text {. }
$$

Here $I_{i j k l}^{D}=\frac{1}{2}\left(\delta_{i k} \delta_{j l}+\delta_{i l} \delta_{j k}\right)-\frac{1}{3} \delta_{i j} \delta_{k l}$ is the fourth order unit deviatoric tensor, $G_{C R}=\eta_{C R} / \Delta t$ is the effective visco-elastic shear modulus, and $G_{P L}=\alpha_{P L} G_{C R}$ is the effective elasto-visco-plastic shear modulus. The non-dimensional tensor $\hat{g}_{k l}$ is given in Appendix B.

We infer from our numerical experiments that anisotropic stress terms in the tangent operator (Eq. 58) have little influence on the convergence rate of the Newton-Raphson iteration. Therefore, we suggest that they can be omitted whenever necessary. With this assumption, the effective elasto-visco-plastic shear modulus $\left(G_{P L}\right)$ becomes essentially equivalent to the effective viscosity (see e.g. Eq. 37 of Moresi et al., 2003 or Fig. 4 of Fullsack, 1995).

Both these papers employ the fixed point method or direct (Picard) iteration (e.g. Zienkiewicz and Taylor, 2000) as a nonlinear solver. Even omitting differences in the tangent operator, there are two substantial differences between the Newton-Raphson and Picard methods:

(i) The Picard method has only external forces and stresses from the previous time step in the right-hand-side, while the Newton-Raphson method has complete out-of-balance 
forces.

(ii) The Picard method operates on total velocity (incremental displacement), while the Newton-Raphson method operates on iterative corrections.

We have tried both Picard and Newton-Raphson methods. Fig. 5 shows a comparison between the two solvers for the shear band initiation benchmark problem, discussed in the next section. We calculated an identical setup by both methods with a gradually increasing number of increments in which we applied a constant amount of extension. Fig. 5 shows the severe sensitivity of the Picard method to increment size. At the same time, the Newton-Raphson method produces accurate results even for comparatively large increments. Other authors (e.g. Muhlhaus and Regenauer-Lieb, 2005) have also pointed out superiority of the Newton-Raphson method over the Picard method.

At the current development stage, we use the Preconditioned Conjugate Gradient (PCG) iterative method (Hestenes, Stiefel, 1952) for both the mechanical and thermal linear systems (Eq. 51 \& Eq.52). For the mechanical system we adopt a symmetric version of the Incomplete LU factorization with Threshold (ILUT) preconditioner (Saad, 1994), equipped with near-optimal nested dissection ordering (George, 1973). The thermal system is preconditioned by the inverse of diagonal of thermal tangent matrix.

The above algorithm, in general, is not numerically scalable (Farhat et al., 2000), i.e. the number of iterations grows nonlinearly with increasing size of the linear system. At present the algorithm can only run in sequential mode. However, it works well with the roughly $10^{5}$ grid nodes in typical 3D regional-scale models on ordinary PCs with 2 GB RAM.

Note that the linear solver may fail in the low-viscosity domains (e.g. asthenosphere), where effective the visco-elastic modulus becomes many orders of magnitude lower than the bulk modulus. We treat this issue by supplementing the mechanical linear solver with the fictitious compressibility technique (e.g. Zienkiewicz and Taylor, 2000). The fictitious bulk modulus is made at maximum two orders of magnitude larger than the shear modulus in the integration point. The algorithm then iteratively removes spurious volumetric strains until the original continuity equation is satisfied.

\subsection{Regridding and Remapping}

After the Newton Raphson step, we resolve the advection terms of the flow. This step is implemented by means of regridding and remapping. The computational sequence of adopted procedure is summarized in the second part of Table 2 .

We employ a specific type of the Arbitrary Lagrangian Eulerian kinematical formulation (Hirt et al., 1997). It is characterized by using a structured Cartesian grid whose nodes are only allowed to move in the vertical direction. With this flexibility, we track the free surface or certain material interfaces inside the model domain. Using the same mechanism we implement erosion and sedimentation processes on the free surface. The bottom surface of the grid is equipped with the Winkler boundary condition.

Lateral boundaries of the grid may by either fixed in space or move parallel to the coordinate axis with constant or variable velocity. This is a particularly useful feature, since it creates a flexible computation window which can track some portion of space, or even stretch or contract laterally. When the length of the mesh in a certain direction changes by the size of the original element, we add (or remove) an entire vertical slice of the elements. Our solvers are designed to 

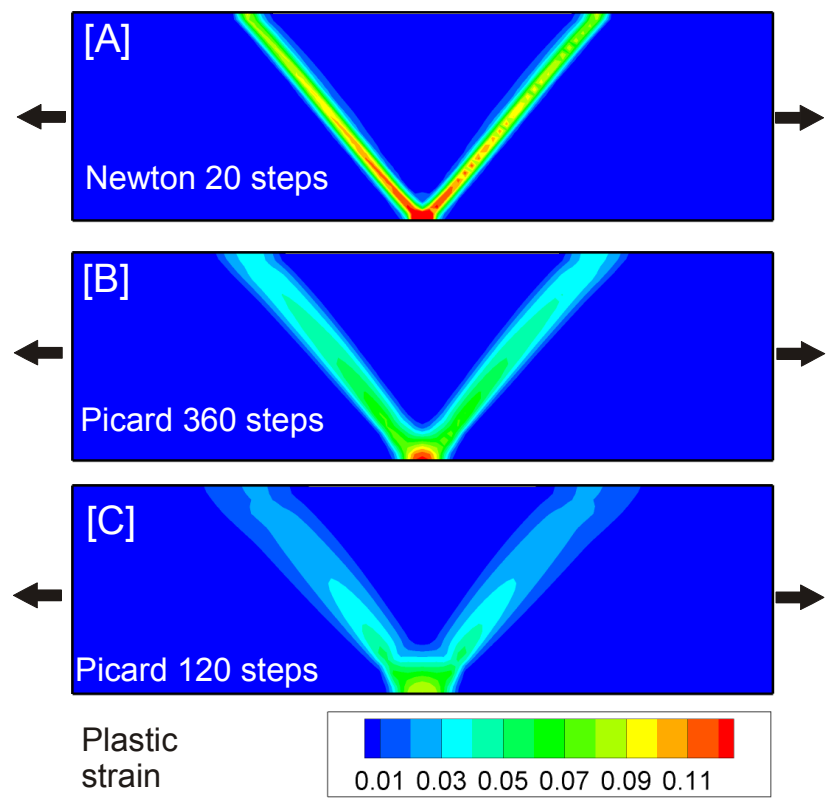

Figure 5 Contour plots of accumulated plastic strain for the shear band initiation problem (see section 4); (a) calculated by Newton-Raphson solver in 20 increments, (b) calculated by Picard solver in 360 increments, (c) calculated by Picard solver in 120 increments. The same amount of extension is applied in all models. In case (a) the shear band has minimum possible width constrained by the cell size. In cases (b) and (c) the shear band is significantly wider and more diffuse slowly decreasing its width with increasing number of time increments.

treat a variable number of elements during computation.

To track material properties and solution history we use the Particle-in-Cell method (Harlow and Welsh, 1965). Each marker has an individual history, including coordinates, material number, pressure, deviatoric stress tensor, accumulated plastic strain, total displacements, and temperature. During the Newton-Raphson step, the history accumulates in cell centers. When markers are advected by mesh, all markers that belong to the cell obtain the same increment of history. When markers are mapped back to the mesh, both material properties and history are averaged among all markers in the updated cell. This incremental approach minimizes spurious numerical diffusion.

We advect markers in the displacement field by consistent interpolation from the mesh. Since the mesh remains rectangular in horizontal plain, the mapping of markers to updated cell positions becomes particularly simple. Certain difficulties exist in regions where a population of markers becomes too dense or too sparse (Moresi et al., 2003). We treat this issue by inserting or deleting markers in cells where the total number of markers falls beyond the limits. We treat material fluxes on the boundaries with the same methodology. When a marker leaves the domain, we simply delete it. When a marker is generated in the boundary cell it acquires material properties and solution history from the closest neighbors. We suggest, therefore, that material extends beyond all boundaries of the domain except the free surface.

We note that the remapping procedure inevitably produces perturbations in the global force balance. We treat these perturbations as initial residuals to be removed on the next time step. This explicit approach to a certain extent reduces the accuracy of the numerical solution (stresses, displacements). Nevertheless, it is common in geodynamics to use similar techniques with the argument that the time step can be reduced if higher accuracy in the time integration is 
required (e.g. Moresi et al. 2003). In section 4.2 we demonstrate that the remapping procedure does not significantly affect the accuracy of the velocity field, which is estimated analytically, even in the benchmark with hundreds of remapping cycles.

\section{Benchmarks and examples}

In this section, we present five simplified problems to verify computer implementation and applicability of the adopted physical models and numerical techniques. The following problems are included:

(i) Bending of an elastic plate

(ii) Sinking of a rigid cylinder in a viscous fluid

(iii) Initiation of shear bands in the brittle crust

(iv) Triaxial compression test

(v) Lithospheric transpressional deformation

The first two problems are designed to test the code's ability to separately handle elastic and viscous rheological mechanisms in $2 \mathrm{D}$. In the first problem, the $2 \mathrm{D}$ formulation (plane strain) is chosen to facilitate comparison with the analytical solution, while in the second problem, the choice is based on the resolution issue. With the sequential solver and without mesh adaptation, the solution of the free-falling Stokes sphere problem with the same resolution would require too much computation time. The next two problems demonstrate quasi-brittle elasto-plastic strain localization in 2D and 3D, respectively. The final problem deals with the complete elasto-viscoplastic rheology in a typical 3D lithospheric-scale setup. Since the plain strain formulation is not currently available in SLIM3D, we discretize entire 2D setups by a single layer of hexahedral elements with zero velocity in the out-of-plane direction. Wherever necessary we introduce and track markers in the mid-surfaces of the elements.

\subsection{Bending of an elastic plate}

We consider bending of an elastic plate which has infinite length in one of the lateral dimensions. The setup of the problem is depicted in Fig. 6, and the geometry, loading and elastic constants of the plate are summarized in Table 4. Similar to a cantilever beam, the plate has one end fixed and the other end free. It has slightly negative buoyancy relative to surrounding material, which allows it to sink and bend. Since we are interested in modeling elastic deformation of the plate, we exclude the surrounding viscous material. Calculations are done in the Lagrangian mode without remeshing.

In this benchmark, we compare the maximum vertical deflection of the plate and the maximum bending stress with the analytical solution of the Euler-Bernoulli beam equation (see Appendix C). Application of this equation requires displacements and thickness to be small compared with length of the plate. We fulfill this requirement by appropriately selecting parameters of the setup. Assuming validity of the Euler-Bernoulli approximation, the maximum deflection of plate can be expressed in terms of parameters in Table 4 as:

$w=\frac{3 \Delta \rho g l^{4}}{2 E h^{2}}$.

For comparison with the numerical solution, we calculate analytical bending stresses using the following relation (see Appendix C): 


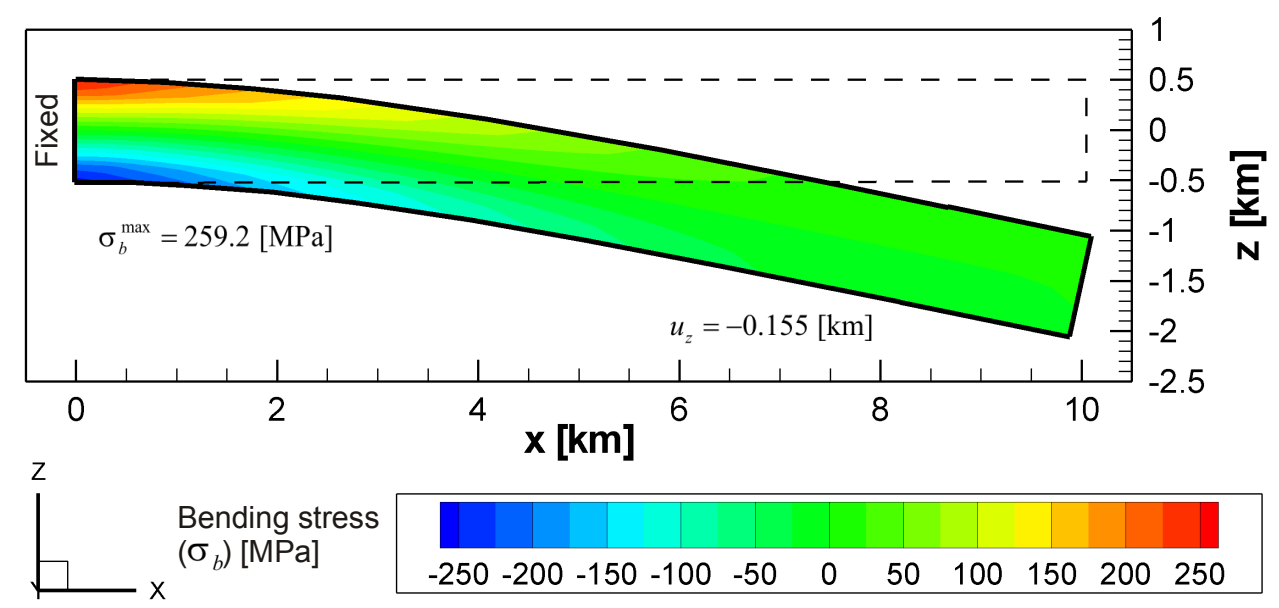

Figure 6 Initial (dashed line) and deformed (solid line) shape of the elastic plate. Vertical displacements are exaggerated by a factor of 10 . Contour plot shows distribution of normal bending stress. Shown are calculated values for maximum bending stress and vertical displacement.

Table 4

Plate benchmark

\begin{tabular}{llll}
\hline Parameter & Meaning & Value & Dimension \\
\hline$\Delta \rho$ & Differential density & 100 & $\mathrm{~kg} \cdot \mathrm{m}^{-3}$ \\
$E$ & Young's modulus & 990 & $\mathrm{kbar}$ \\
$l \times h$ & Size of the domain & $10 \times 1$ & $\mathrm{~km}$ \\
$n_{l} \times n_{h}$ & Discretization & $35 \times 7$ & $\mathrm{elements}$ \\
$w_{\text {theor }}$ & Theoretical deflection & 0.151 & $\mathrm{~km}$ \\
$w_{\text {calc }}$ & Calculated deflection & 0.155 & $\mathrm{~km}$ \\
$e r r_{w}$ & Rel. deflection error & 2.6 & $\%$ \\
$\sigma_{\text {theor }}$ & Theoretical stress & 257.2 & $\mathrm{MPa}$ \\
$\sigma_{\text {calc }}$ & Calculated stress & 259.2 & $\mathrm{MPa}$ \\
$e r r_{\sigma}$ & Rel. stress error & 0.8 & $\%$ \\
$\sigma_{\text {cycl }}$, & Cyclic stress residual & 0.1 & $\%$ \\
\hline
\end{tabular}

$\sigma_{b}= \pm \frac{3 \Delta \rho g l^{2}(h-\Delta h)}{h^{2}}$

where $\Delta h$ is the element size along the height of the beam.

We apply and remove the gravity load multiple times in this model. Each time, when the load is removed, we allow the plate to restore its original shape and simultaneously release stored elastic stresses. During the solution, we monitor spurious residual stresses, which tend to accumulate during multiple load cycles. This deficiency is related to the rate formulation of elasticity adopted in SLIM3D (see Eq. 8). Unlike exact large strain elasticity models (e.g. Bonet and Wood, 1997), this formulation produces nonzero dissipation of elastic energy along the closed deformation path (e.g. Belytschko et al., 2000). The applicability of the model to rocks is based on the fact that elastic strains remain small, while elastic stresses constantly dissipate either by plastic or viscous rheological mechanisms. In this benchmark, we numerically assess spurious dissipation of elastic energy. 


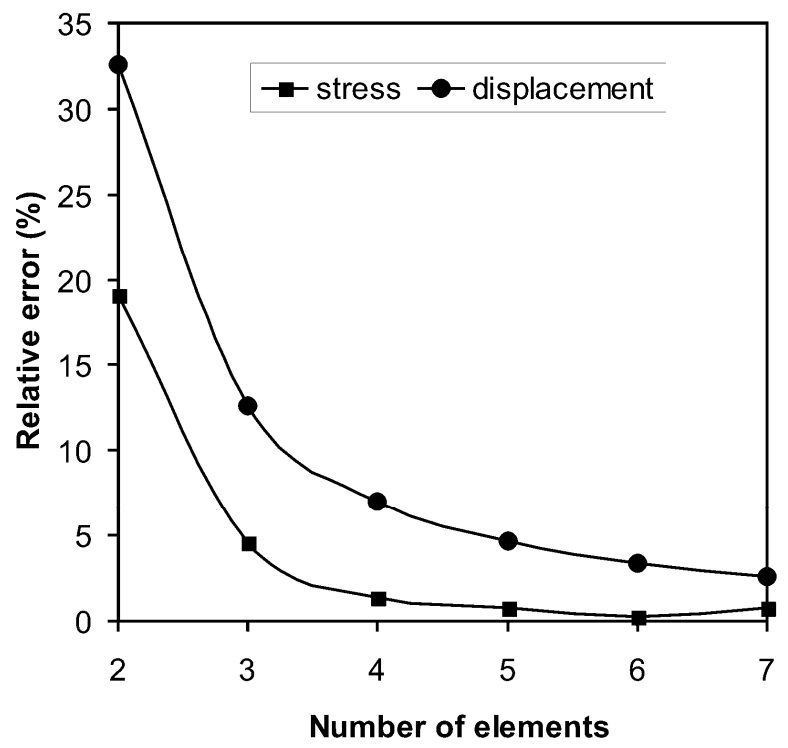

Figure 7 Relative error in approximation of stresses (rectangles) and vertical displacements (circles) versus number of elements across the elastic plate thickness.

Fig. 6 shows the shape of the deformed plate (exaggerated by factor of 10) along with a contour plot of normal bending stresses. Key results of the benchmark are presented in Table 4. We investigate influence of discretization on the accuracy of the solution using different numbers of elements along the plate thickness starting with just two elements (since we have a single integration point per element). In all cases, the $1: 2$ (thickness vs. length) element aspect ratio is maintained within the vertical plane. Fig. 7 shows the relative error in the approximation of stresses and displacements versus number of elements along the plate thickness. Note that discretization converges relatively fast, providing acceptable accuracy (around 5.0\% error) with just 4-5 elements across the plate. The results in Table 4 correspond to discretization by 7 elements across the plate. Further increasing the number of elements did not yield a substantial increase in the accuracy in neither the vertical deflection $(2.6 \%$ error) nor in the bending stresses $(0.8 \%$ error $)$. We attribute this fact partially to inaccuracies of the approximate analytical solution and partially to the presence of shear locking (e.g. Belytschko et al., 2000) of the employed elements. After 10 cycles of cyclic loading, the residual stress remains about $0.1 \%$ of peak bending stress $(0.3 \mathrm{MPa}$ vs. $300.0 \mathrm{MPa})$. Thus, we conclude that the stress integration algorithm has sufficient accuracy for practical applications.

\subsection{Sinking of a rigid cylinder in a viscous fluid}

The purpose of this benchmark is twofold: (i) we test the ability to simulate purely viscous flow with large abrupt changes of the viscosity; (ii) we simultaneously assess the quality of the remeshing algorithm (treatment of markers and numerical diffusion issue). Setup of the problem includes one half of a rectangular domain which is $800 \mathrm{~km}$ wide and $700 \mathrm{~km}$ deep (see Fig. 8) occupied by viscous fluid. We impose sticking between the domain walls and the fluid. On the left side of the domain we impose a symmetry condition. An infinite cylinder with $25 \mathrm{~km}$ radius is initially placed at $250 \mathrm{~km}$ depth. Due to negative buoyancy the cylinder sinks into the fluid. We maintain relative rigidity of the cylinder by a 4 orders of magnitude viscosity contrast with the surrounding fluid. Parameters of the setup are summarized in Table 5. Numerical solution is obtained with a time step $10^{4}$ years.

In this benchmark, we compare analytical and numerical estimation of the cylinder velocity. The 
[A] $0.25 \mathrm{Myr}$

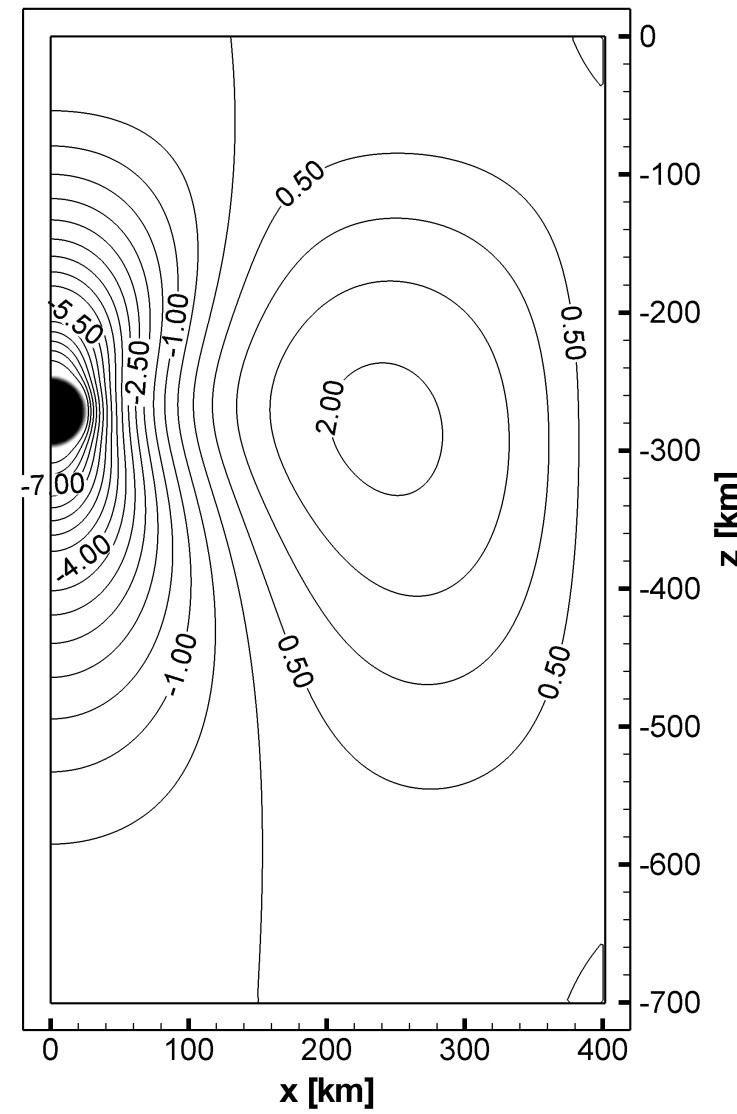

[B] $2.25 \mathrm{Myr}$

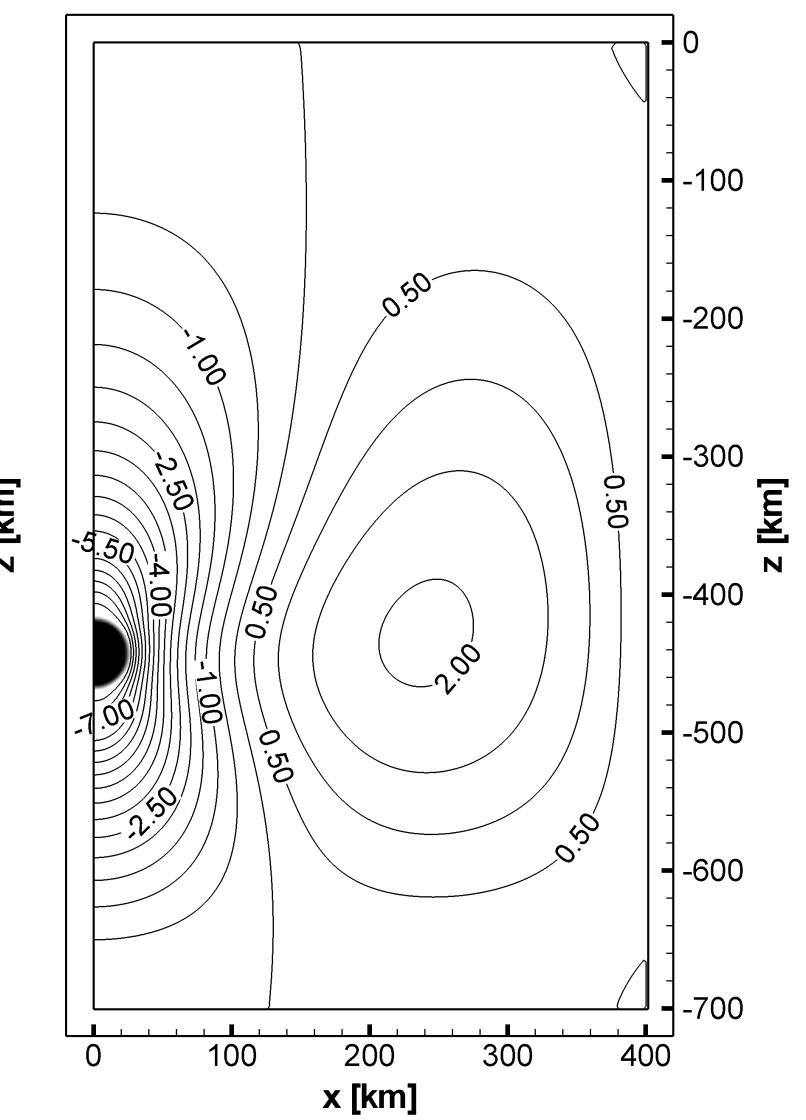

Figure 8 Isolines of vertical velocity for the cylinder problem. (a) after $0.25 \mathrm{Myr}$, (b) after $2.25 \mathrm{Myr}$. Black circles indicate positions of the particles which represent the cylinder.

analytical solution for resisting force due to motion of the cylinder in a reservoir of finite size can be found in Slezkin (1955). By equating resistance force with Archimedes force one can obtain the following expression for the velocity of the cylinder (see Appendix D):

$v_{c}=\frac{1}{4}\left(\ln k-\frac{k^{2}-1}{k^{2}+1}\right) \frac{r^{2} \Delta \rho g}{\eta_{f}}, \quad k=\frac{b}{r}$,

where $\eta_{f}$ is fluid viscosity, $r$ is the radius of cylinder and $b$ is the characteristic distance between the cylinder and rigid wall of the domain. In our setup this distance is equal to $375 \mathrm{~km}$.

We plot the distribution of vertical velocity together with positions of cylinder markers at two different times in Fig. 8 (see also Animation 1 in the online version of the paper). Note that through the multiple remeshing steps, cylinder shape shows completely no sign of numerical diffusion. The cylinder remains perfectly circular and undisturbed through the course of computation. We therefore conclude an acceptable quality of adopted remeshing algorithm. The average velocity of the cylinder constitutes around $8.5 \mathrm{~cm}$ per year. The maximum relative difference between the analytical and numerical estimations is less than $1.7 \%$ (see Table 5). Error in the numerical solution for the velocity field remains relatively low, despite hundreds of remapping cycles and a large time step ( $10^{4}$ years). Increase of viscosity contrast up to 7 orders of magnitude did not result in any substantial changes in the numerical solution. We conclude that the adopted numerical formulation allows handling of purely viscous flow with large abrupt variations of the viscosity. 
Table 5

Cylinder benchmark

\begin{tabular}{llll}
\hline Parameter & Meaning & Value & Dimension \\
\hline$\Delta \rho$ & Differential density & 100 & $\mathrm{~kg} \cdot \mathrm{m}^{-3}$ \\
$r$ & Radius of cylinder & 25 & $\mathrm{~km}$ \\
$l \times h$ & Size of the domain & $400 \times 700$ & $\mathrm{~km}$ \\
$n_{l} \times n_{h}$ & Discretization & $200 \times 350$ & $\mathrm{elements}$ \\
$\eta_{f}$ & Viscosity of fluid & $10^{20}$ & $\mathrm{~Pa} \cdot \mathrm{s}$ \\
$\eta_{c}$ & Viscosity of cylinder & $10^{24}$ & $\mathrm{~Pa} \cdot \mathrm{s}$ \\
$v_{\text {theor }}$ & Theoretical velocity & 8.47 & $\mathrm{~cm} \cdot \mathrm{yr}^{-1}$ \\
$v_{\text {calc }}$ & Calculated velocity & 8.61 & $\mathrm{~cm} \cdot \mathrm{yr}^{-1}$ \\
\hline
\end{tabular}

Table 6

Shear bands benchmark

\begin{tabular}{llll}
\hline Parameter & Meaning & Value & Dimension \\
\hline$\rho$ & Density & 3000 & $\mathrm{~kg} \cdot \mathrm{m}^{-3}$ \\
$K$ & Bulk modulus & 630 & $\mathrm{kbar}$ \\
$G$ & Shear modulus & 400 & $\mathrm{kbar}$ \\
$\varphi$ & Friction angle & 30 & $\left(^{\circ}\right)$ \\
$\psi$ & Dilatation angle & 0 & $\left(^{\circ}\right)$ \\
$c$ & Cohesion & 20 & $\mathrm{MPa}$ \\
$l \times h$ & Size of the domain & $40 \times 7$ & $\mathrm{~km}$ \\
$n_{l} \times n_{h}$ & Discretization & $200 \times 35$ & $\mathrm{elements}$ \\
$\alpha_{\text {ext }}$ & Inclination angle (extension) & 53 & $\left(^{\circ}\right)$ \\
$\alpha_{\text {comp }}$ & Inclination angle (compression) & 38 & $\left(^{\circ}\right)$ \\
\hline
\end{tabular}

\subsection{Initiation of shear bands in the brittle crust}

Formation of zones of localized shear (shear bands) during planar deformation has been the subject of extensive research (see e.g. Arthur et al., 1977; Muhlhaus and Vardoulakis, 1987; Vermeer, 1990; Borja and Aydin, 2004; Buiter et al., 2006; Kaus and Podladchikov, 2006). One of the most important questions concerns preferred orientation of the shear bands. Three basic models are generally discussed (Coulomb, 1773; Roscoe, 1970; and Arthur et al., 1977). For a pressure-sensitive material with friction angle $\varphi$ and dilatation angle $\psi$, these models predict the following angles between the shear band and direction of minor principal stress:

$$
\begin{array}{ll}
\alpha=45^{\circ}+\varphi / 2 & \text { (Coulomb), } \\
\alpha=45^{\circ}+\psi / 2 & \text { (Roscoe), } \\
\alpha=45^{\circ}+(\varphi+\psi) / 4 & \text { (Arthur). }
\end{array}
$$

The theoretical analysis for the onset of shear banding presented by Vermeer (1990) also yields the Roscoe and Coulomb angles as limits for possible shear-band orientation. However, due to elastic unloading the shear band can, in principle, assume any orientation within this range.

In this benchmark, we tested the capability to model initiation of shear bands and compare inclination angles of modeled shear bands with analytical estimations and numerical solutions obtained by other codes. Since only the onset of shear bands is considered, calculations are done 

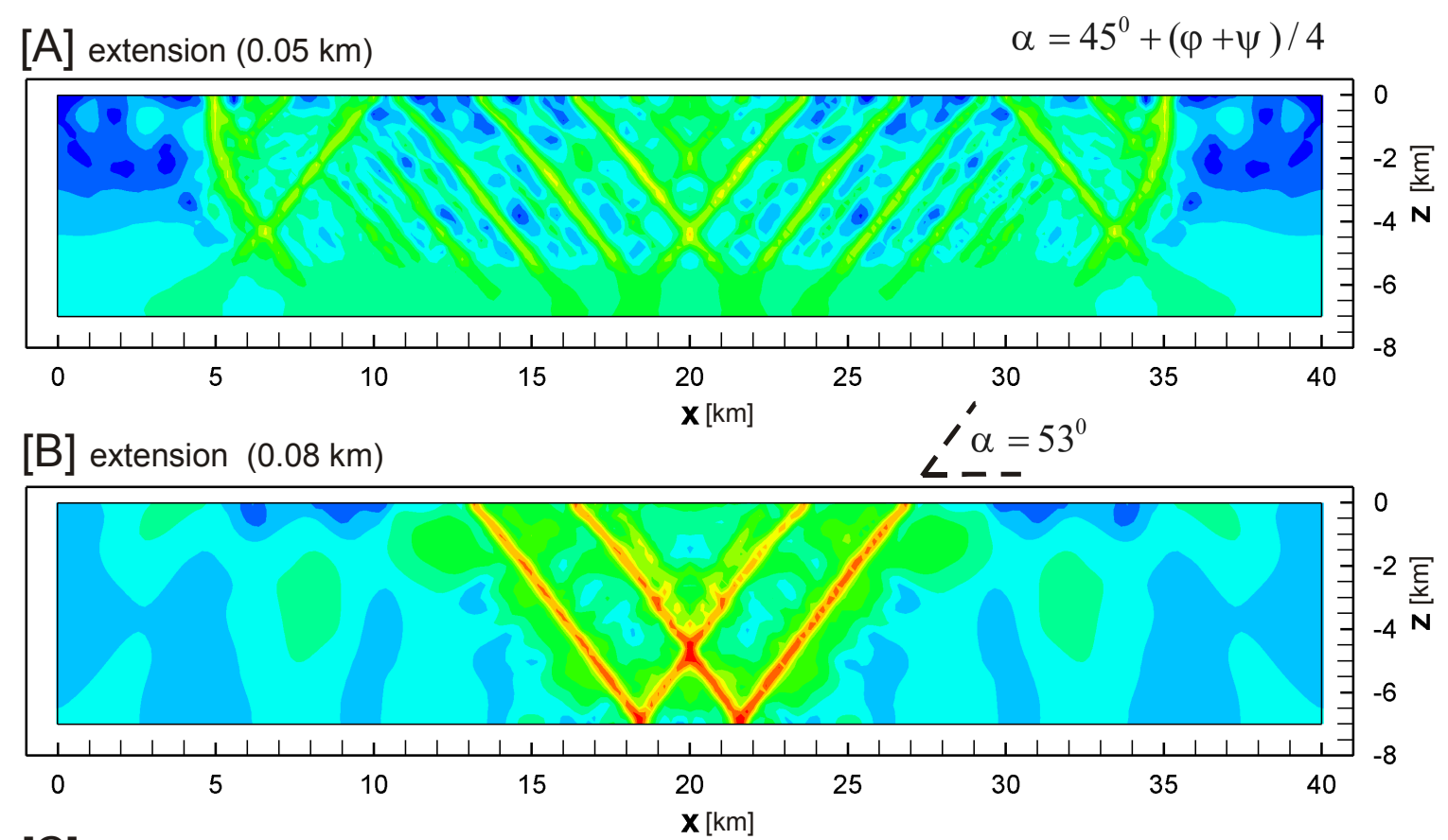

[C] compression $(0.15 \mathrm{~km})$

$\alpha=45^{0}-(\varphi+\psi) / 4$
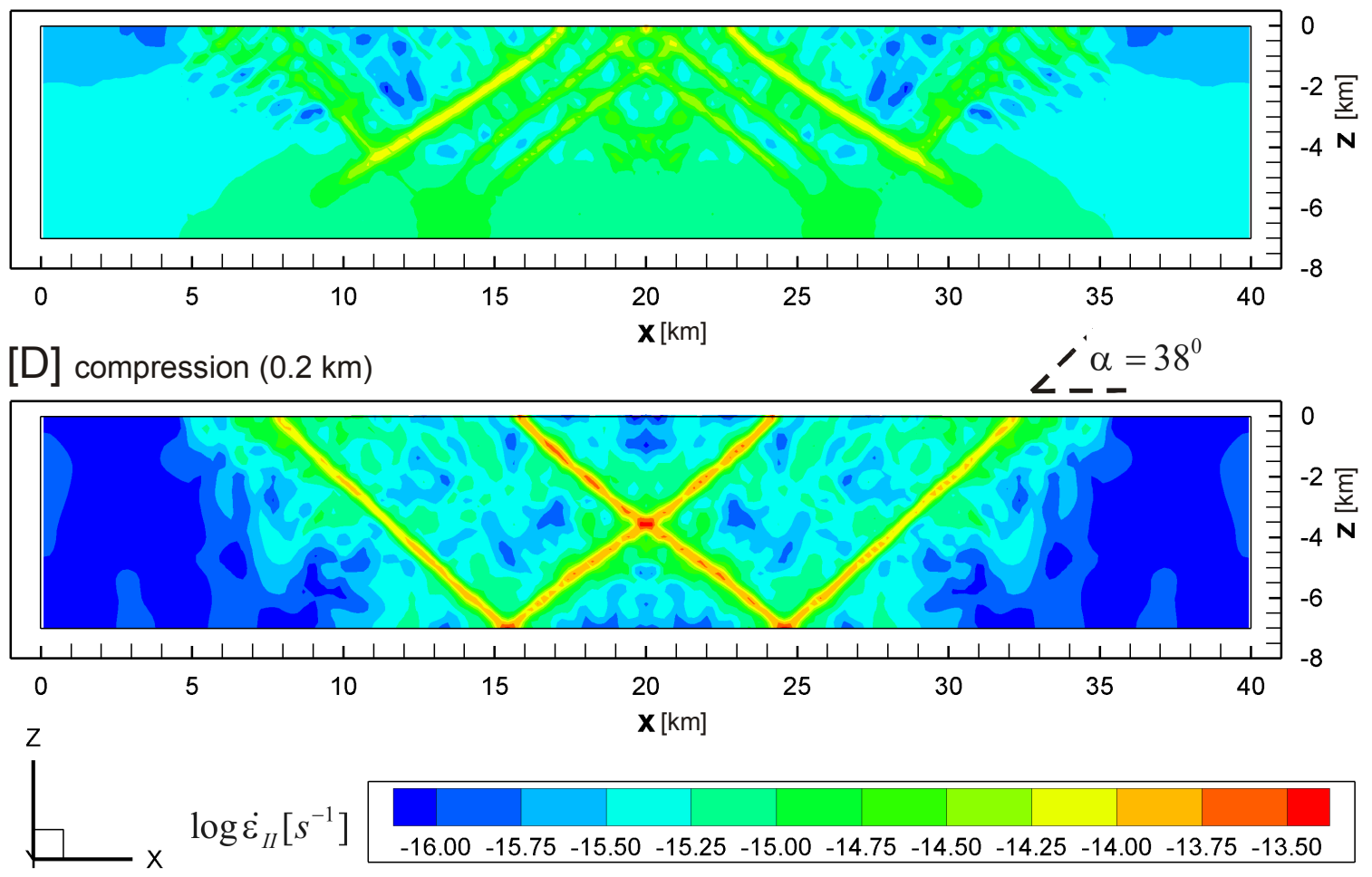

Figure 9 Contour plot of effective logarithmic strain rate for the shear band initiation problem. (a) $0.05 \mathrm{~km}$ of extension, (b) $0.08 \mathrm{~km}$ of extension, (c) $0.15 \mathrm{~km}$ of compression (d) $0.2 \mathrm{~km}$ of compression. Dashed lines indicate expected orientation of shear bands according to Arthur et al. (1977) (expression shown in the figure). Also shown are the values of Arthur angles between the shear bands and horizontal axis.

in the small strain regime. The large strain plastic localization in 3D is additionally demonstrated in the next subsection.

The model setup includes a crustal domain which is $40 \mathrm{~km}$ wide and $7 \mathrm{~km}$ thick (see Fig. 9). On 
the top boundary we impose a free surface, while the bottom boundary has zero normal velocity and tangential free-slip. We consider two loading cases. On the lateral boundaries, we prescribe $1 \mathrm{~cm}$ per year normal velocity such that it causes either shortening or extension of the domain. We assume an elasto-plastic rheology typical of mafic crust (see Table 6). Since we employ the purely deviatoric Prandtl-Reuss flow rule, the dilatation angle is zero in our formulation.

We measure inclination angles of the shear bands from the horizontal axis. In the case of compression the orientation of the minor principal stress is vertical. Therefore the angle with respect to horizontal axis should be computed with a minus sign; for example, the Coulomb angle should be $\alpha=45^{\circ}-\varphi / 2$. In the case of extension, the minor principal stress is horizontal; therefore sign should be plus i.e. $\alpha=45^{\circ}+\varphi / 2$.

Fig. 9 shows the contour plot of effective strain rate for both extension $(a, b)$ and compression (c, d) cases at different stages of the process (see also Animation 2 in the online version of the paper). The shear bands start to propagate from the surface where strength is minimal. We note that it takes significantly less extension than shortening for shear bands to cut the entire domain. The initial pattern of shear bands is highly chaotic. Some of them have inclination angles close to Coulomb angles $\left(30^{\circ}, 60^{\circ}\right)$ at this stage. At later stages, only a few shear bands survive. They begin to accommodate entire deformation caused by shortening / extension such that other shear bands become completely inactive. The mature post-failure shear bands precisely match the Arthur angles $\left(38^{\circ}, 53^{\circ}\right)$. In both cases (compression or tension), shear bands at the Roscoe angles $\left(45^{\circ}\right)$ did not occur in the model.

Our results are in good agreement with results of the other codes. For example, Poliakov and Herrmann (1994) solved a similar problem using a non-associative Mohr-Coulomb rheology and explicit FLAC technique. In their solution, the shear bands formed spontaneously and assumed various inclination angles ranging between the Roscoe and Coulomb limits. The results obtained with I2ELVIS (Gerya and Yuen, 2007) for the numerical sandbox experiment (Buiter et al., 2006) revealed that the majority of shear bands in the numerical solution tend to assume the Arthur angle. We conclude that our code is able to effectively simulate origination and propagation of localized shear zones.

\subsection{Triaxial compression test}

Elasto-plastic strain localization in the large strain regime is further demonstrated by numerical simulation of the triaxial compression test. We consider a laterally unconfined parallelepiped specimen of size $1 \times 1 \times 3 \mathrm{~km}$ discretized into $20 \times 20 \times 60$ hexahedral elements, subjected to kinematical axial compressional loading (Fig. 10a and Fig. 11a). Material parameters are assumed from the previous benchmark (see Table 6). We specify two different types of heterogeneity: either small random seeds uniformly distributed over the specimen (Fig. 10a) or an oblique gash located near the edge as shown in Fig. 11a. In both cases, localization is induced by relatively low cohesion of the seeds with respect to surrounding material (down to $20 \%$ of nominal).

The type of heterogeneity significantly impacts the pattern of deformation observed in the model. Thus, the random seeding induces formation of multiple shear bands as shown in Figs. $10 \mathrm{~b}-10 \mathrm{~d}$ (see also Animation 3 in the online version of the paper). Despite that the specimen exhibits fragmentation into small pieces, one can easily distinguish major failure plane, which gradually evolves though the course of loading. In contrast, the gash-type seeding immediately 
[A]

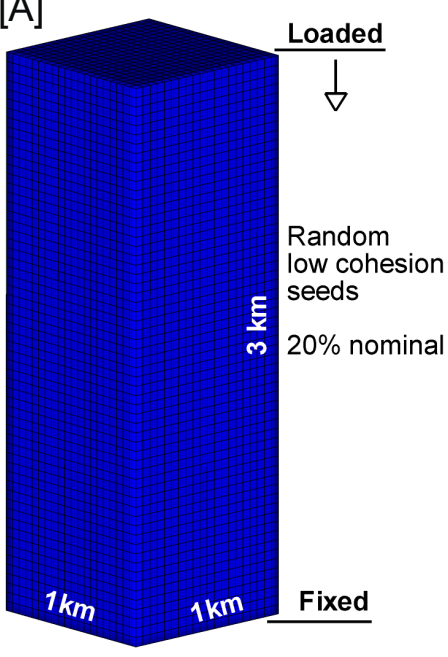

[B]

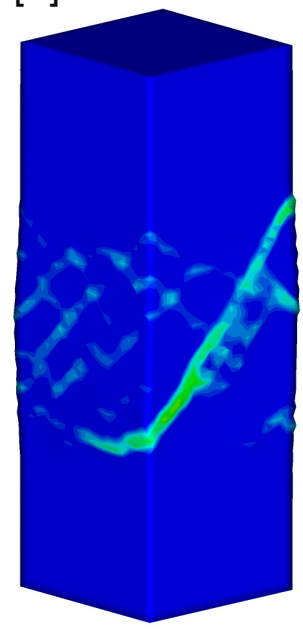

[C]

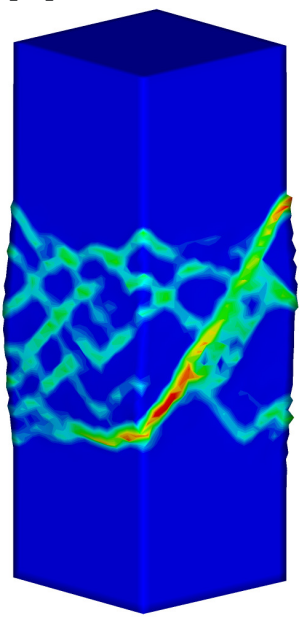

[D]

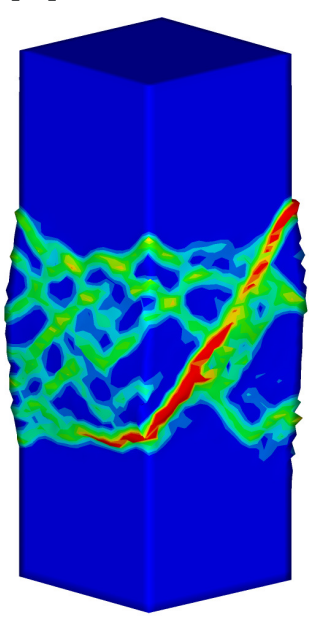

Z

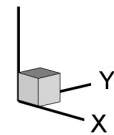

Plastic strain

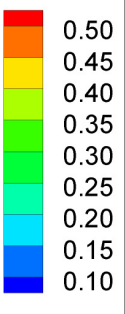

Figure 10 Setup (a) and results $(b-d)$ of the triaxial compression numerical test with uniform random seeding (see text). Cases $\mathrm{b}-\mathrm{d}$ correspond to $2.5 \%, 4.2 \%, 5.8 \%$ of axial deformation, respectively. Magnitude of accumulated plastic strain is shown in colors.
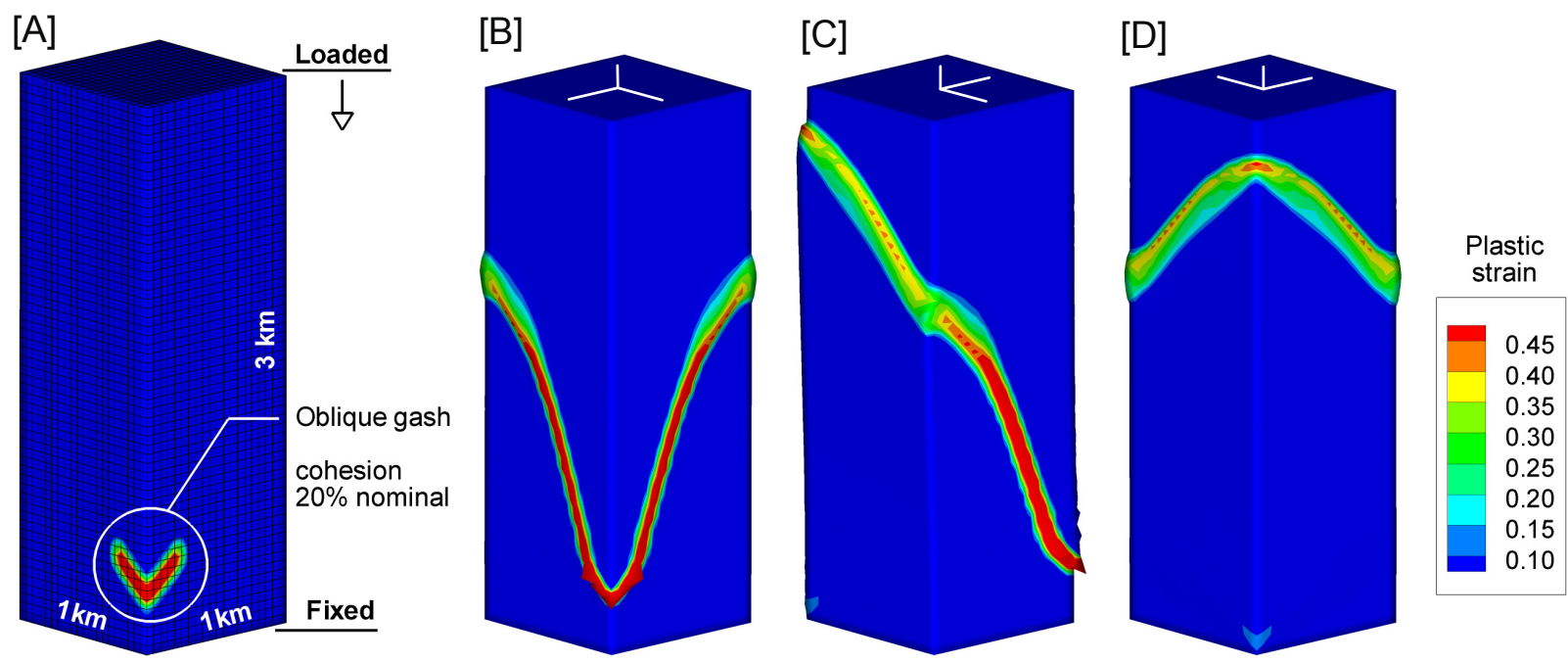

Figure 11 Setup (a) and results $(b-d)$ of the triaxial compression numerical test with oblique gash seeding (see text). Cases $b-\mathrm{d}$ differ by amount of specimen rotation around vertical axis $\left(0^{\circ}, 90^{\circ}\right.$ and $180^{\circ}$, respectively) and all correspond to $2.7 \%$ of axial deformation. White lines on the top face show coordinate axis for rotational reference. Magnitude of accumulated plastic strain is shown in colors.

produces a complex curvilinear failure plane, which separates the entire specimen into two parts (Figs. 11b-11d). The majority of the shear band inclination angles also coincide with the Arthur angles. Results of the triaxial compression test support the conclusions of previous benchmark for the 3D case and the large strain regime.

\subsection{Lithospheric transpressional deformation}

In this example, we present a typical 3D lithospheric-scale setup which includes effects of elastovisco-plastic rheology with a temperature controlled brittle-ductile transition. The model consists of a lithospheric domain which has lateral dimensions 200 by $600 \mathrm{~km}$ and depth $80 \mathrm{~km}$, as shown in Fig. 12a. The domain is discretized into $36 \times 108 \times 16$ hexahedral elements (68561 grid points). Compositional heterogeneity includes equally thick $(15 \mathrm{~km})$ felsic upper crust and mafic 
Table 7

Rheological and thermal parameters used in transpression problem

\begin{tabular}{lllll}
\hline Parameter & Dimension & $\begin{array}{l}\text { Felsic } \\
\text { Upper Crust }\end{array}$ & $\begin{array}{l}\text { Mafic } \\
\text { Lower Crust }\end{array}$ & $\begin{array}{l}\text { Mantle } \\
\text { peridotite }\end{array}$ \\
\hline$\rho$ & $\mathrm{g} \cdot \mathrm{cm}^{-3}$ & 2.7 & 3.0 & 3.3 \\
$K$ & $\mathrm{kbar}$ & 550 & 630 & 1220 \\
$G$ & $\mathrm{kbar}$ & 360 & 400 & 740 \\
$\log B_{N}$ & $\mathrm{~Pa}^{-n} \cdot \mathrm{s}^{-1}$ & -28.0 & -21.05 & -16.3 \\
$H_{N}$ & $\mathrm{~kJ} \cdot \mathrm{mol}^{-1}$ & 223 & 445 & 535 \\
$n$ & - & 4.0 & 4.2 & 3.5 \\
$\varphi$ & $\left({ }^{\circ}\right)$ & 30 & 30 & 30 \\
$c$ & $\mathrm{MPa}$ & 20 & 20 & 20 \\
$\alpha$ & $\mathrm{K}-1$ & $2.7 \cdot 10^{-5}$ & $2.7 \cdot 10^{-5}$ & $3.0 \cdot 10^{-5}$ \\
$C_{p}$ & $\mathrm{~kJ} \cdot \mathrm{kg}^{-1} \cdot \mathrm{K}^{-1}$ & 1.2 & 1.2 & 1.2 \\
$\lambda$ & $\mathrm{W} \cdot \mathrm{m}^{-1} \cdot \mathrm{K}^{-1}$ & 2.5 & 2.5 & 3.3 \\
$A$ & $\mathrm{nW} \cdot \mathrm{kg}^{-1}$ & 1.0 & 0.1 & 0.0 \\
\hline
\end{tabular}

lower crust and a $50 \mathrm{~km}$ thick peridotite mantle. Rheological and thermal parameters used in the modeling are presented in Table 7. At elongated vertical boundaries we impose zero normal velocities as well as $3.5 \mathrm{~cm} /$ year of right-lateral strike-slip velocity. The upper and lower boundaries are the free surface and the Winkler support boundary, respectively. At the short vertical boundaries we impose lithostatic pressure along with a free-slip condition.

The initial temperature distribution is shown in Fig.12b. We use different temperature gradients in the upper crust, and the lower crust with lithospheric mantle to account for radiogenic heat. In the asthenosphere, we assume a temperature of $1300^{\circ} \mathrm{C}$. The lithosphere-asthenosphere boundary is initially placed at $70 \mathrm{~km}$ depth. To initiate the state of transpression in the model, we use a zone of slightly thinned lithosphere (10 km of thinning), which is oriented obliquely to the strike-slip direction.

Fig. 12c shows a contour plot of the effective strain rate on the free surface and in a typical crosssection after $3 \mathrm{Myr}$ of evolution and $105 \mathrm{~km}$ of strike-slip (see also Animation 4 in the online version of the paper). The far-field strike-slip deformation tends to localize in a narrow zone located above the initially thinned lithosphere where strength is minimal. This mechanism leads to the self-consistent formation of the boundary between two lithospheric plates. The structure of the plate boundary changes with depth from a very narrow shear zone in the brittle upper crust to progressively more diffuse and wide shear zone in the ductile lower crust and upper mantle. The brittle upper crust tends to form regular secondary structures (en-echelon faults). Fig. 12d shows a contour plot of material phases and isolines of vertical displacements. The transpressional character of strike-slip deformation leads to surface uplift on pressure ridges related to enechelon structures as well as corresponding crustal thickening along the entire plate boundary.

Fig. 12e shows the influence of temperature and stress on the effective viscosity distribution. The overall viscosity variation in this model is about 7 orders of magnitude, with most of this variation caused by gradual temperature increase with depth and compositional heterogeneities. Among the local effects, we note the effects of shear heating and nonlinear creep which come into play across the entire plate boundary. Relative stress peaks associated with both the upperlower crust boundary and the Moho, commonly referred to as "christmas-trees" (e.g. Brace and Kohlstedt 1980), can be recognized in Fig. 12f. Note that due to strain softening, the strength of 
[A]

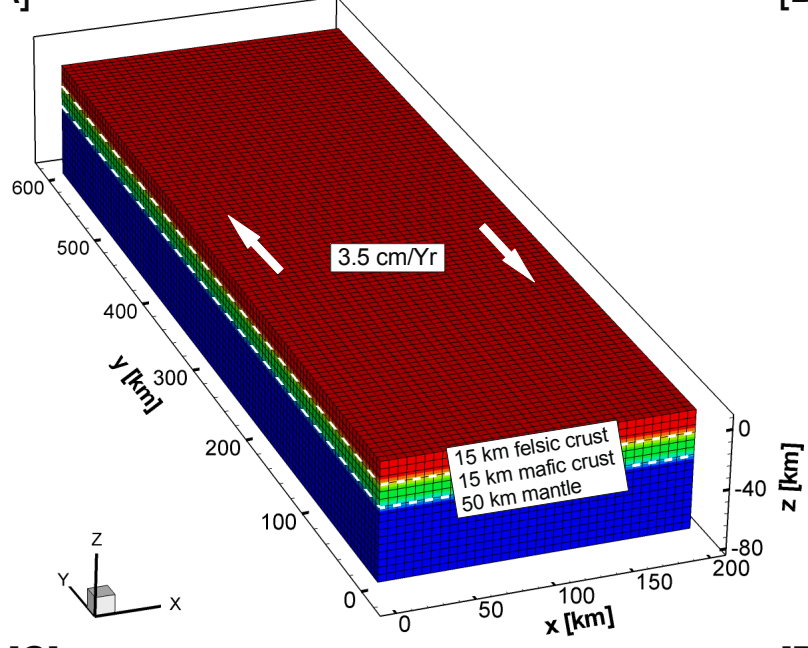

[C]

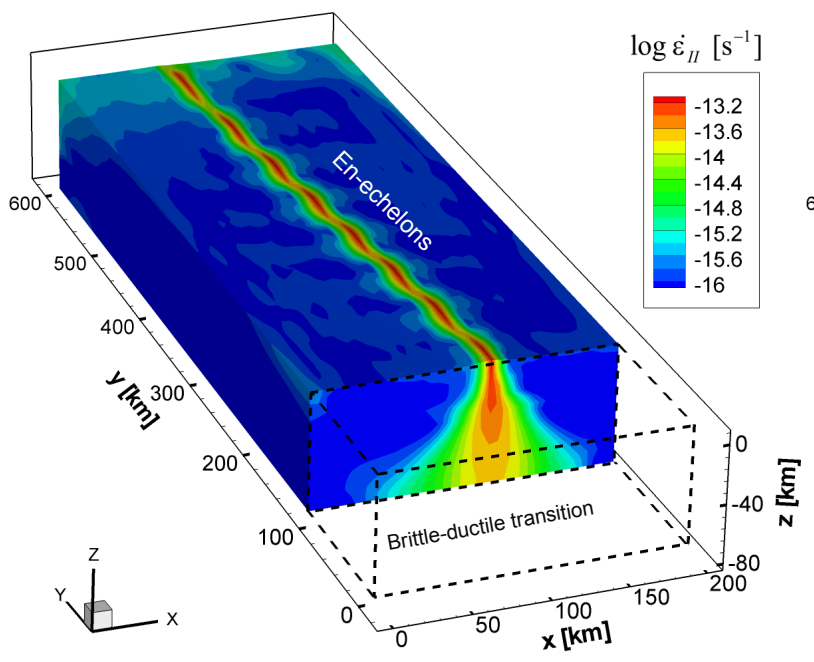

[B]

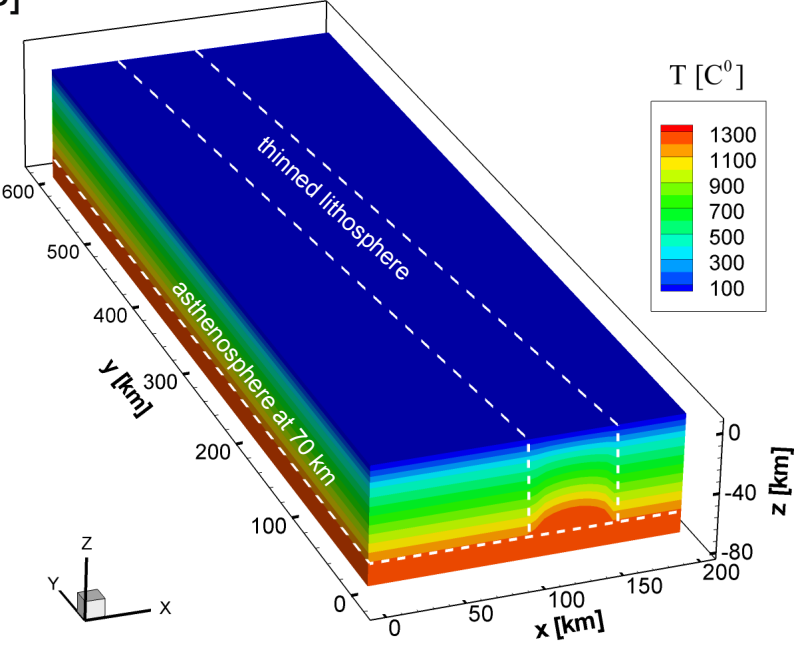

[D]

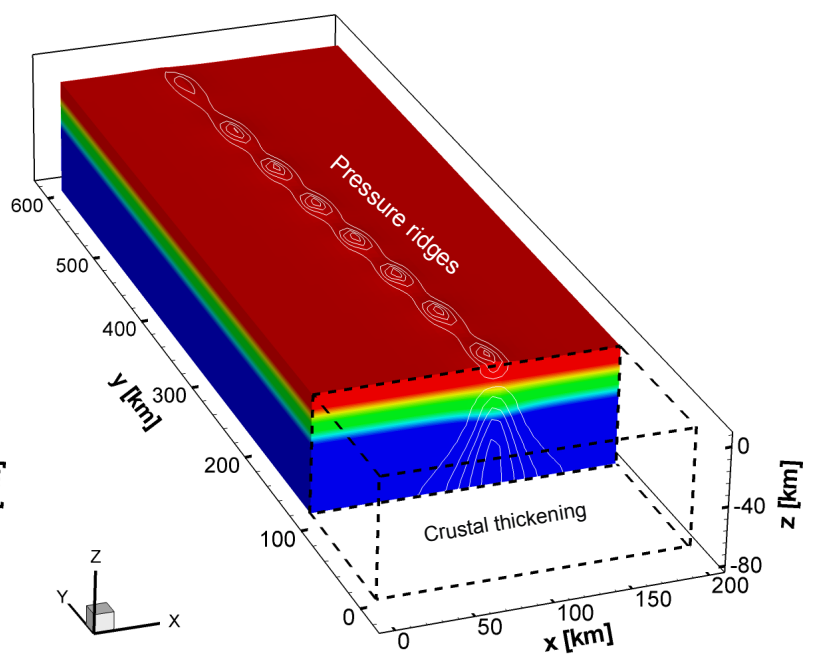

[E]

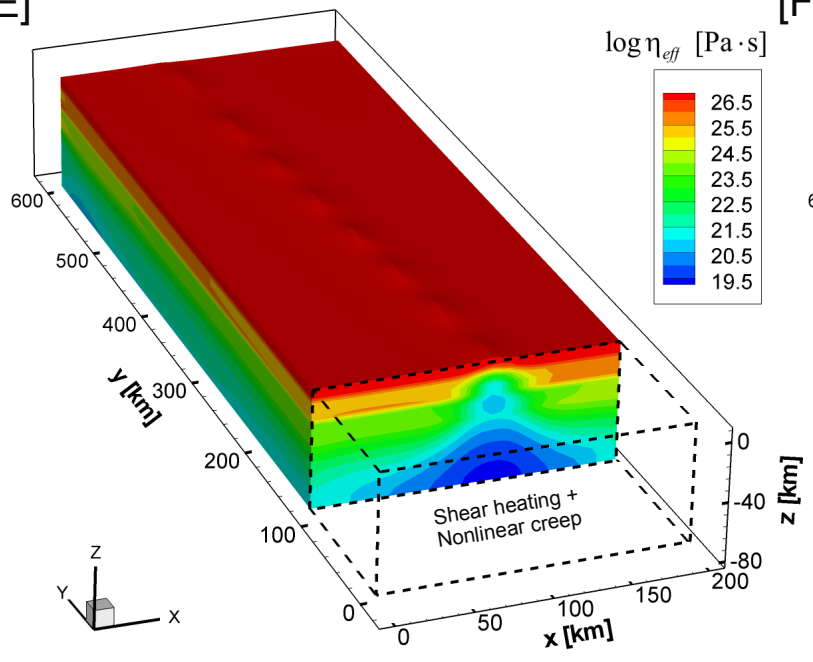

$[\mathrm{F}]$

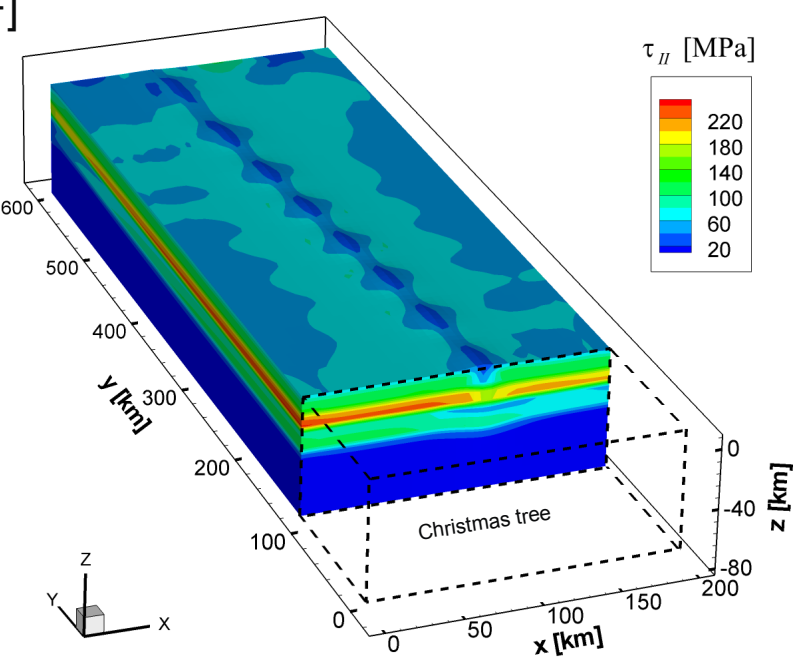

Figure 12 Lithospheric transpressional deformation problem. (a) setup and discretization, (b) initial thermal model, (c) distribution of effective strain rate on the surface and in the cross-section after 3Myr, (d) material phases (colors) and vertical displacements (white lines), (e) distribution of effective logarithmic viscosity (f) effective stress. 

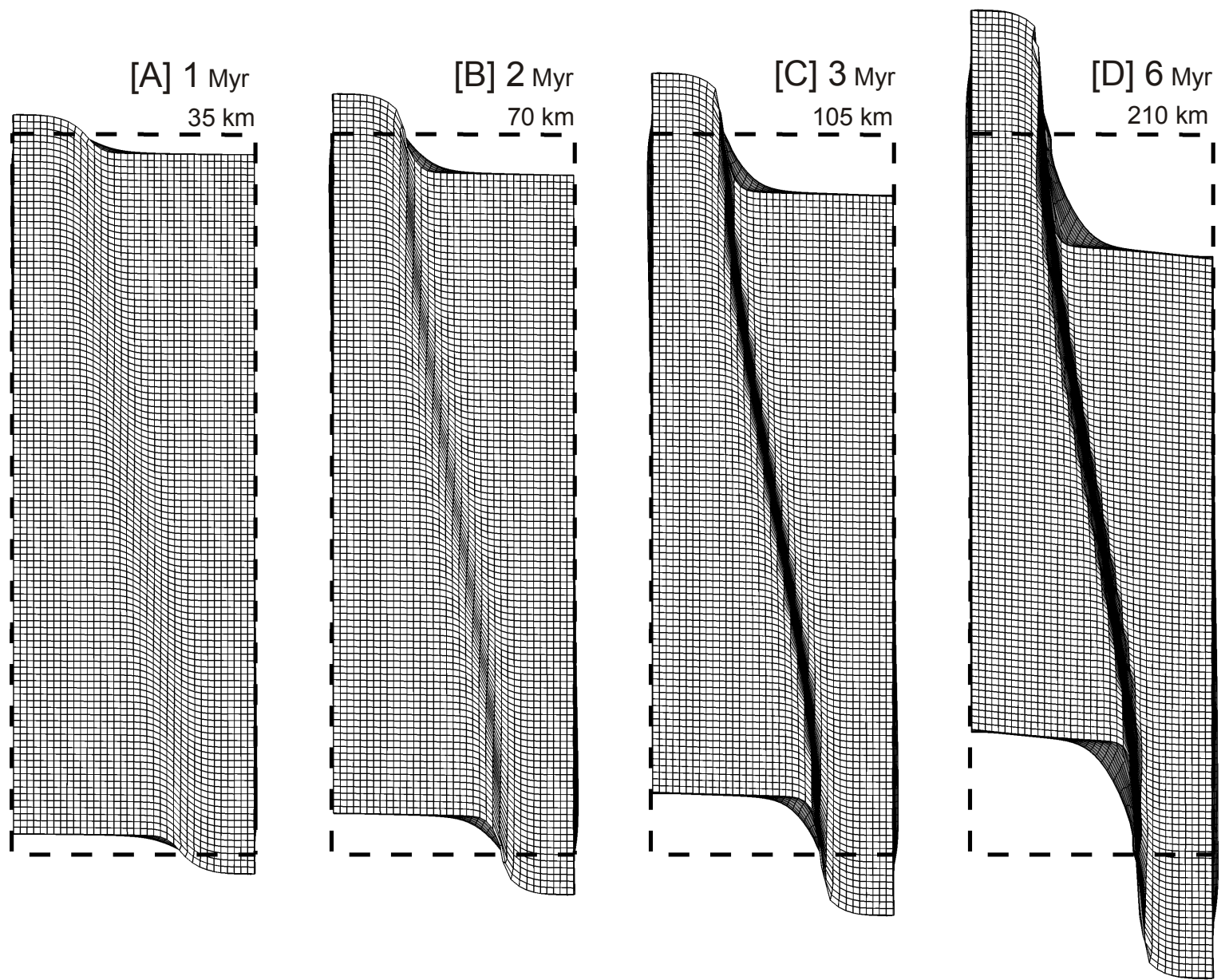

Figure 13 Evolution of the Lagrangian mesh embedded in the material for the lithospheric transpressional deformation problem. View from the top of the model. Thick dashed lines show the lateral boundaries of calculation domain shown in Fig. 12. Note that material fluxes on the boundaries of the domain are nonzero.

major faults becomes low, which is also visible from the stress distribution on the surface (Fig. $12 \mathrm{f})$.

Fig. 13 show a 6 Myr evolution of the Lagrangian mesh embedded in the material (see also Animation 5 in the online version of the paper). We note that material fluxes on the boundary of computational box are nonzero. Incoming material acquires the properties and solution history from the closest material inside the box. The magnitude of the strike-slip displacement is large $(210 \mathrm{~km})$ and comparable to the characteristic size of the model. The transpressional plate boundary accumulates significant shear strains during the evolution. Summarizing these results we suggest that our code, in principle, can be applied for 3D modeling of geological scale evolution of lithospheric plate boundaries.

\section{Summary and discussion}

\subsection{What is next?}

Currently, the code SLIM3D already includes many techniques necessary for modeling deformation processes on lithospheric and geologic scales. Namely, we have implemented 
coupled thermo-mechanical solutions, realistic elasto-visco-plastic rheology, and a kinematic framework that is able to handle large deformations. However, in this section we discuss the techniques which are still missing in the code. We also point out the directions we plan to develop our code to satisfy the growing needs of the new modeling section in GFZ-Potsdam.

The major disadvantage is that code is still severely restricted in problem size. This fact is explained mostly by the following: (i) the code is still sequential (ii) the code utilizes a linear solver which is not numerically scalable (Farhat et al., 2000). To remove these disadvantages, we plan to extend our code in the following mainstream directions of linear solver technology:

(i) Direct parallel multifrontal solvers (Gupta et al., 1997; Amestoy et al., 2000)

(ii) Multigrid methods with adaptation for plasticity (Adams, 2000; Ekevid et al., 2004)

(iii) Dual-primal domain decomposition methods (Farhat et al., 2000)

At the same time, we are planning to parallelize the entire finite element routines using the MPI package.

Deformation processes in the lithosphere occur on highly variable spatial scales. To sufficiently resolve the material length scale in lithospheric models, the element size should be of the order of hundreds of meters (Regenauer-Lieb and Yuen, 2003). Therefore, a successful code for lithospheric-scale modeling should allow variable spatial resolution. In SLIM3D we are presently restricted to a structured non-adaptive grid. A possible way to introduce adaptivity is to switch to unstructured tetrahedral mesh generators (Rassineux, 1998) or octree-based methods (Shephard et al., 1991; Braun et al., this issue). These will be investigated in the future.

Apart from advancements in numerical methods, we are planning to further develop physical models of rocks introducing advanced damage rheological models and incorporating metamorphic reactions and melting as well as coupled porous flow. Furthermore, we plan to introduce coupling between deformation processes at regional and global spatial scales as well as at seismic-cycle and geological time scales.

\subsection{Summary remarks}

This paper presents a new tool (SLIM3D) for three-dimensional lithospheric-scale modeling. The code includes a coupled thermo-mechanical treatment of deformation process and allows complicated elasto-visco-plastic rheology with diffusion, dislocation and Peierls creep and Mohr-Coulomb plasticity. The code incorporates an Arbitrary Lagrangian Eulerian formulation with free surface and Winkler boundary conditions.

We have developed practical implementation of elasto-visco-plastic rheology and shown that Maxwell time does not pose an upper limit for the integration time step in the numerical scheme.

We have applied the full Newton-Raphson method for solution of discrete balance equations and demonstrated its superiority over the Picard method, which is widely used in geodynamics.

For plasticity model we have obtained simplified expressions for the tangent operator. Anisotropic stress terms in tangent operator have little influence on the convergence rate of the Newton-Raphson iteration. Therefore they can be omitted whenever necessary. With this assumption, the difference between effective viscosity and tangent operator concepts vanishes.

With linear sequential solvers, which are not numerically scalable, we are able to treat problems with up to hundred thousand of grid points on ordinary PCs. It is expected that capabilities of the 
method can be drastically expanded by implementing numerically scalable parallel solvers (e.g. multigrid) as well as an adaptive mesh.

\section{Acknowledgments}

This work was funded by Deutsche Forschungsgemeinschaft (DFG) grant So 425/2 in the framework of the International Continental Scientific Drilling Program (ICDP) and GeoForschungsZentrum Potsdam. We greatly appreciate constructive and helpful reviews offered by Stefan Schmalholz and an anonymous reviewer. Anton Popov heartily thanks Alex Vlasov and Mike Mnushkin for multiple productive discussions regarding conceptual aspects of the development.

\section{Appendix A. Plastic stress correction}

We recall the spectral decomposition of Cauchy stress tensor (e.g. Borja et al. 2003):

$$
\sigma_{i j}=\sum_{A=1}^{3} \sigma_{A} m_{i j}^{(A)}, \quad m_{i j}^{(A)}=\widehat{n}_{i}^{(A)} \widehat{n}_{j}^{(A)},
$$

where $\sigma_{A}$ denotes principal stresses (negative in compression), $m_{i j}^{(A)}$ are the correspondent spectral direction tensors, and $\hat{n}_{i}^{(A)}$ are the principal direction vectors.

Deviatoric-volumetric stress decomposition holds in principal stress space as well, therefore we can write:

$\sigma_{A}=\tau_{A}-p$,

where $\tau_{A}$ are the principal deviatoric stresses.

The Mohr-Coulomb yield surface can be rewritten using (A2) as follows:

$F=\frac{1}{2}\left(\tau_{\max }-\tau_{\min }\right)+\frac{1}{2}\left(\tau_{\max }+\tau_{\min }\right) \sin \varphi-p \sin \varphi-c \cos \varphi \leq 0$.

The plastic stress correction according to the Prandtl-Reuss flow rule reduces to the simple radial stress return (e.g. Wilkins, 1964). Practical implementation consists of the following proportional scaling of trial principal deviatoric stresses:

$\tau_{A}=\alpha_{P L} \tau_{A}^{t r}$,

where $0<\alpha_{P L}<1$ is the plastic scaling ratio, which can be directly determined by substituting (A4) into the yield surface expression (A3) and enforcing the condition $F=0$. The result is:

$\alpha_{P L}=\frac{2(p \sin \varphi+c \cos \varphi)}{(1+\sin \varphi) \tau_{\max }^{t r}-(1-\sin \varphi) \tau_{\min }^{t r}}$.

According to the Prandtl-Reuss flow rule, the updated stress deviator remains co-axial with the trial stress deviator, therefore we can update directly (omitting spectral decomposition) as:

$\tau_{i j}=\alpha_{P L} \tau_{i j}^{t r}$.

Using the identities:

$\tau_{I I}=\alpha_{P L} \tau_{I I}^{t r}, \quad \tau_{I I}=\tau_{I I}^{t r}-2 \eta_{C R} \dot{\gamma}$,

we can readily solve for the magnitude of plastic strain rate: 
$\dot{\gamma}=\frac{1}{2 \eta_{C R}}\left(1-\alpha_{P L}\right) \tau_{I I}^{t r}$

\section{Appendix B. Linearization}

To complete formulation of the Newton-Raphson method, it is necessary to obtain an expression for the tangent matrices $\partial \mathbf{f} / \partial \Delta \mathbf{u}$ and $\partial \mathbf{w} / \partial \mathbf{T}$. Unfortunately, in general, it poses a difficult algebraic task. The resulting expressions are always non-symmetric except for trivial cases (Simo and Hughes, 2000). Moreover, in geodynamical modeling it is necessary to simulate nonlinear effects like erosion, sedimentation or melt emplacement, which often cannot be linearized analytically. Numerical differentiation may overcome this difficulty (see e.g. PerezFoguet et al., 2000). In this paper, however, we admit certain assumptions to obtain approximate linearization, which still possesses acceptable convergence properties.

First, we assume that the integrals in the discrete balance equations Eq. (23) \& Eq. (24) are configuration-independent. This assumption substantially simplifies linearization and avoids additional non-symmetry in the tangent matrices. In this case, the linearization of the energy equation becomes trivial. The result reads:

$E_{I J}=\frac{\partial w_{I}}{\partial T_{J}}=\int_{\Omega} N_{I} \frac{\rho C_{p}}{\Delta t} N_{J} d \Omega+\int_{\Omega} \frac{\partial N_{I}}{\partial x_{i}} \lambda \frac{\partial N_{J}}{\partial x_{i}} d \Omega$.

We note that during Newton-Raphson iteration, all integrals in the above equation are evaluated over latest available coordinates.

Linearization of internal stress term yields:

$$
\frac{\partial}{\partial \Delta u_{k J}}\left(\frac{\partial N_{I}}{\partial x_{j}} \sigma_{i j}\right)=\frac{\partial N_{I}}{\partial x_{j}}\left(C_{i j k l}+L_{i j k l}\right) \frac{\partial N_{J}}{\partial x_{l}} \text {. }
$$

The term $C_{i j k l}=\partial \sigma_{i j} / \partial \widehat{\varepsilon}_{k l}$ is referred to as the (material) tangent operator (Simo \& Taylor 1985). The term $L_{i j k l}=\partial \sigma_{i j} / \partial \omega_{k l}$ can be called the rotation tangent operator. Here $\widehat{\varepsilon}_{k l}$ denotes the total strain increment in contrast with the deviatoric strain increment $\varepsilon_{k l}$, which appears in Eq. (32). Recalling the expression for trial deviatoric stress (Eq. 36), the rotation operator can be expanded as follows:

$$
L_{i j k l}=\frac{\partial \sigma_{i j}}{\partial \omega_{k l}}=\frac{\partial}{\partial R_{p q}}\left(\alpha_{C R} R_{i m}{ }^{n} \tau_{m n} R_{j n}\right) \frac{\partial R_{p q}}{\partial \omega_{k l}} .
$$

After straightforward, but quite cumbersome algebra, which we omit here (http://matrixcookbook.com), one obtains the following expression for the rotation tangent operator:

$L_{i j k l}=\frac{1}{4} \alpha_{C R}\left(W_{i k} Q_{j l}+Q_{i l} W_{j k}-W_{i l} Q_{j k}-Q_{i k} W_{j l}\right)$,

where the auxiliary tensors are given by:

$W_{i j}=\left(\delta_{i j}-\frac{1}{2} \omega_{i j}\right)^{-1}, Q_{i j}=R_{i k} \sigma_{k l}\left(R_{j l}+\delta_{j l}\right)$.

Expression (B4) lacks major symmetry i.e. $L_{i j k l} \neq L_{k l i j}$. Moreover, we note that it becomes identically zero in the viscous case since $\alpha_{C R} \rightarrow 0$.

We note that a similar approach was presented e.g. by Fish and Shek (1999). They rigorously linearized all geometrical nonlinearities related to the Hughes-Winget scheme together with configuration dependence. From their results, we conclude that potential advantages from 
completely consistent linearization are still marginal, taking into account additional burdens related to the solution of non-symmetric systems. Therefore, we suggest omitting the rotation operator from the tangent matrix approximation. In the case that a non-symmetric solver is available, one can use the unmodified expression (B4). Alternatively, some kind of symmetrization can be employed, for example:

$L_{i j k l}^{\mathrm{sym}}=\frac{1}{2}\left(L_{i j k l}+L_{k l i j}\right)$.

The material tangent operator $C_{i j k l}=\partial \sigma_{i j} / \partial \widehat{\varepsilon}_{k l}$ forms two cases depending on type of the flow, either visco-elastic or elasto-visco-plastic. It is convenient to decompose the material tangent operator into volumetric and deviatoric parts. Applying the chain rule we can write:

$C_{i j k l}=\frac{\partial \sigma_{i j}}{\partial \widehat{\varepsilon}_{k l}}=\frac{\partial \tau_{i j}}{\partial \varepsilon_{m n}} \frac{\partial \varepsilon_{m n}}{\partial \widehat{\varepsilon}_{k l}}-\delta_{i j} \frac{\partial p}{\partial \theta} \frac{\partial \theta}{\partial \widehat{\varepsilon}_{k l}}$.

First, we consider a nonlinear visco-elastic deformation process. In this case, we use expressions for updated pressure (Eq. 35) and trial deviatoric stress (Eq. 36). Motivated by the moderate stress dependence of the effective creep viscosity, we assume $\partial \eta_{C R} / \partial \tau_{i j}=0$ to avoid cumbersome differentiation of the expressions (9) - (12) together with expressions (37). The resultant material tangent operator related to nonlinear visco-elastic deformation reads:

$C_{i j k l}=K \delta_{i j} \delta_{k l}+2 G_{C R} I_{i j k l}^{D}$.

Here, $I_{i j k l}^{D}$ is the fourth order unit deviatoric tensor:

$I_{i j k l}^{D}=\frac{1}{2}\left(\delta_{i k} \delta_{j l}+\delta_{i l} \delta_{j k}\right)-\frac{1}{3} \delta_{i j} \delta_{k l}$,

and $G_{C R}$ is the effective shear modulus of visco-elastic creep:

$G_{C R}=\eta_{C R} / \Delta t$.

We note that $G_{C R}$ has appropriate viscous and elastic limits given by:

$\lim _{\alpha_{C R} \rightarrow 0} G_{C R}=\eta_{e f f} / \Delta t, \quad \lim _{\alpha_{C R} \rightarrow 1} G_{C R}=G$.

Next, we consider the elasto-visco-plastic deformation process. Recalling the plastic stress correction formula (Eq. 42) and applying the chain rule, we expand the deviatoric term in the following form:

$$
\frac{\partial \tau_{i j}}{\partial \widehat{\varepsilon}_{k l}}=\underbrace{2 G_{P L} I_{i j k l}^{D}}_{\text {isotropic }}+\underbrace{\tau_{i j}^{t r} \widehat{g}_{k l}}_{\text {anisotropic }},
$$

where $G_{P L}$ is the effective elasto-visco-plastic shear modulus (similar to the corresponding effective viscosity; see e.g. Moresi et al., 2003; Fullsack, 1995)

$G_{P L}=\alpha_{P L} G_{C R}$,

and $\hat{g}_{k l}$ is non-dimensional second order tensor which is given by:

$\widehat{g}_{k l}=\frac{\partial \alpha_{P L}}{\partial \varepsilon_{m n}} I_{m n k l}^{D}$.

We note that the elasto-visco-plastic tangent operator has both isotropic and anisotropic components. The latter destroys major symmetry similar to the rotation operator, so that the nonsymmetric linear solver is again required. From our numerical experiments we infer that symmetric isotropic approximation (the first term in Eq. B12) possesses acceptable convergence properties together with the full Newton-Raphson method. Therefore, we suggest that the anisotropic term can be either dropped out or symmetrized in a standard way (see Eq. B6).

For completeness, we derive the anisotropic stiffness terms in this Appendix. Recalling the 
expression for plastic scaling ratio (Eq. 43) and invoking the chain rule once again, we perform the following expansion:

$$
\frac{\partial \alpha_{P L}}{\partial \varepsilon_{m n}}=2 G_{C R}\left(\frac{\partial \alpha_{P L}}{\partial \tau_{\min }^{t r}} \frac{\partial \tau_{\min }^{t r}}{\partial \tau_{m n}^{t r}}+\frac{\partial \alpha_{P L}}{\partial \tau_{\max }^{t r}} \frac{\partial \tau_{\max }^{t r}}{\partial \tau_{m n}^{t r}}\right) .
$$

Entire derivatives in the above equation are readily available. For convenience, we denote:

$$
D=\frac{(p \sin \varphi+c \cos \varphi)}{\left[(1+\sin \varphi) \tau_{\text {max }}^{t r}-(1-\sin \varphi) \tau_{\min }^{t r}\right]^{2}},
$$

then derivatives of plastic scaling ratio can be expressed as:

$$
\begin{aligned}
& \alpha_{\text {min }}=\frac{\partial \alpha_{P L}}{\partial \tau_{\min }^{t r}}=-2(\sin \varphi-1) D, \\
& \alpha_{\text {max }}=\frac{\partial \alpha_{P L}}{\partial \tau_{\max }^{t r}}=-2(\sin \varphi+1) D .
\end{aligned}
$$

The orthogonality of principle directions yields the following expressions for derivatives of principal stresses:

$\frac{\partial \tau_{\min }^{t r}}{\partial \tau_{m n}^{t r}}=m_{m n}^{(\min )}, \quad \frac{\partial \tau_{\max }^{t r}}{\partial \tau_{m n}^{t r}}=m_{m n}^{(\max )}$

where $m_{m n}^{(\min )}$ and $m_{m n}^{(\max )}$ are the spectral direction tensors (see Appendix A) associated with minimum and maximum principal stresses, respectively. Combining the above results and applying deviatoric projection (see Eq. B14), we obtain the final expression for non-dimensional tensor:

$\hat{g}_{k l}=2 G_{C R}\left(\alpha_{\min } m_{k l}^{(\min )}+\alpha_{\max } m_{k l}^{(\max )}\right)-\frac{2}{3} G_{C R}\left(\alpha_{\min }+\alpha_{\max }\right) \delta_{k l}$.

Here, we have used the fact that trace of the spectral direction tensor is equal to unity.

Linearization of the Winkler boundary condition yields:

$\frac{\partial}{\partial \Delta u_{k J}}\left(N_{I} \bar{\sigma}_{i j} n_{j}\right)=N_{I} \frac{\partial \bar{\sigma}_{i j}}{\partial \Delta u_{k J}} n_{j}$.

Here we have assumed that the outward normal vector does not vary with displacements. Rewriting the expression for boundary stress tensor (Eq. 22) in incremental form we have:

$\bar{\sigma}_{i j}={ }^{n} \bar{\sigma}_{i j}-\rho_{\text {ext }} g \Delta u_{z} \delta_{i j}$,

where $\Delta u_{z}$ is the vertical component of the displacement increment vector (downward positive). We can write, upon linearization of (B21), the following:

$$
\frac{\partial \bar{\sigma}_{i j}}{\partial \Delta u_{k J}}=-\rho_{e x t} g N_{J} \hat{z}_{k} \delta_{i j} \text {. }
$$

We note that $\hat{z}_{k}$ denote components of unit vector of vertical axis. Substituting (B22) into (B20) and reducing to symmetrical form, we can build the following approximation for the Winkler stiffness term:

$\frac{\partial}{\partial \Delta u_{k J}}\left(N_{I} \bar{\sigma}_{i j} n_{j}\right)=N_{I}\left(-\rho_{\text {ext }} g n_{z} \hat{z}_{i} \hat{z}_{k}\right) N_{J}$.

Here, $n_{z}$ denotes vertical component of outward normal vector.

Finally, the approximate mechanical tangent matrix takes the following form:

$$
K_{i k J J}=\int_{\Omega} \frac{\partial N_{I}}{\partial x_{j}} C_{i j k l} \frac{\partial N_{J}}{\partial x_{l}} d \Omega+\int_{\Gamma} N_{I}\left(\rho_{\text {ext }} g n_{z} \hat{z}_{i} \hat{z}_{k}\right) N_{J} d \Gamma
$$




\section{Appendix C. Cantilever beam}

We consider a cantilever beam of length $l$ with rectangular cross-section of width $b$ and thickness $h$. Normal stress related to bending in the cross-section is generally computed using the following formula (see e.g. Blake, 1985):

$\sigma_{b}=\frac{M}{I} z$.

Here, $M$ is the bending moment, $z$ is the distance between the point where we want to calculate stress and center of the cross-section, and $I$ is the second moment of inertia of the cross-section, which for rectangular shapes is given by:

$I=\frac{1}{12} b h^{3}$.

Maximum compressive and tensile stresses occur on the top and bottom faces of beam where $z= \pm \frac{1}{2} h$. In the benchmark problem, however, we calculate analytical stresses in slightly different locations. The points offset from the top and bottom face by half the size of the element to match location of integration points in the numerical scheme. Thus, we use $z= \pm \frac{1}{2}(h-\Delta h)$, where $\Delta h$ is the element size along the height of beam.

In a cantilever beam loaded by gravity, the maximum bending moment on the fixed end is computed as follows:

$M=\frac{q l^{2}}{2}$,

where $q$ is the weight of unit length of the beam, which is given by:

$q=b h \Delta \rho g$,

where $\Delta \rho$ is the differential density of the beam relative to surrounding material. Combining equations $(\mathrm{C} 1)-(\mathrm{C} 4)$ we obtain an analytical estimation for the bending stress:

$\sigma_{b}= \pm \frac{3 \Delta \rho g l^{2}(h-\Delta h)}{h^{2}}$.

Maximum vertical deflection of the free end of the beam is given by (see e.g. Blake, 1985):

$w=\frac{q l^{4}}{8 E I}$,

where $E$ is Young's modulus of the material. In the case that elastic properties are specified in terms of bulk and shear moduli, the Poisson's ratio and Young's modulus can be expressed, respectively, as follows:

$v=\frac{3 K-2 G}{6 K+2 G}, \quad E=2 G(1+v)$.

In the benchmark problem, we use typical values for mafic crust: $K=630 \mathrm{kbar}, G=400 \mathrm{kbar}$, which roughly gives us the value of Young's modulus $E=990 \mathrm{kbar}$. Substituting equations (C2) and (C4) into (C6), we obtain analytical estimation for maximum vertical deflection of the beam:

$w=\frac{3 \Delta \rho g l^{4}}{2 E h^{2}}$.

\section{Appendix D. Rigid cylinder}

We consider penetration of a rigid cylinder with radius $r$ into a viscous fluid with viscosity $\eta_{f}$. 
An analytical solution for the infinite fluid reservoir does not exist, which is clearly stated in the well-known Stokes paradox (see e.g. Slezkin, 1955). If we assume, however, that the reservoir has a finite characteristic dimension, the analytical solution can be obtained. Resistance force per unit length can be estimated using the following expression (Slezkin, 1955):

$$
R=\frac{4 \pi}{\ln k-\frac{k^{2}-1}{k^{2}+1}} \eta_{f} v_{c}, \quad k=\frac{b}{r},
$$

where $v_{c}$ is the velocity of the cylinder, and $b$ is the distance between cylinder and rigid wall of the reservoir. We note that a sticking boundary condition must be enforced on the reservoir walls.

The steady-state velocity of the cylinder can be estimated by equating resistance force with negative buoyancy force, which is given by:

$F=\pi r^{2} \Delta \rho g$,

where $\Delta \rho$ is the differential density of cylinder relative to surrounding fluid. The resulting expression reads:

$v_{c}=\frac{1}{4}\left(\ln k-\frac{k^{2}-1}{k^{2}+1}\right) \frac{r^{2} \Delta \rho g}{\eta_{f}}$.

\section{References}

Adams, M.F., 2000. Parallel multigrid solvers for 3D unstructured finite element problems in large deformation elasticity and plasticity. Int. J. Numer. Meth. Engng., 48: 1241-1262.

Alejano, L.R., Alonso, E., 2005. Considerations of the dilatancy angle in rocks and rock masses. Int. J. Rock Mech. Min. Sci., 42: 481-507.

Amestoy, P.R., Duff, I.S., L'Excellent, J.-Y., 2000. Multifrontal parallel distributed symmetric and unsymmetric solvers. Comput. Methods Appl. Mech. Engrg., 184: 501-520.

Arthur, J.R.F., Dunstan, T., Al-Ani, Q.A.J., Assadi, A., 1977. Plastic deformation and failure of granular media. Geotechnique, 27: 53-74.

Babeyko, A.Yu., Sobolev, S.V., Trumbull, R.B., Oncken, O., Lavier, L.L., 2002. Numerical models of crustal scale convection and partial melting beneath the Altiplano-Puna plateau. Earth Planet. Sci. Lett., 199: 373-388.

Babuska, I., 1973. The finite element method with Lagrangian multipliers. Num. Math., 20: 179-192.

Bailey, R.C., 2006. Large time step numerical modelling of the flow of Maxwell materials.

Geophys. J. Int., 164: 460-466.

Bathe, K.J., Ramm, E., Wilson, E.L., 1975. Finite element formulations for large deformation dynamic analysis. Int. J. Numer. Meth. Engng., 9: 353-386.

Belytschko, T., Liu, W.K., Moran, B., 2000. Nonlinear Finite Elements for Continua and Structures. John Wiley \& Sons, Chichester.

Belytschko, T., Tabbara, M., 1993. H-adaptive finite element methods for dynamic problems, with emphasis on localization. Int. J. Numer. Meth. Engng., 36: 4245-4265. 
Bercovici, D., 1993. A simple model of plate generation from mantle flow. Geophys. J. Int., 114: 635650.

Blake, A., 1985. Handbook of Mechanics, Materials, and Structures. Wiley, New York.

Bonet, J., Wood, R.D., 1997. Nonlinear Continuum Mechanics for Finite Element Analysis. Cambridge University Press, Cambridge.

Bonet, J., Marriott, H., Hassan, O., 2001. An averaged nodal deformation gradient linear tetrahedral element for large strain explicit dynamic applications. Commun. Numer. Meth. Engng., 17: 551-561.

Borja, R.I., Aydin, A., 2004. Computational modeling of deformation bands in granular media, I: Geological and mathematical framework. Comput. Methods Appl. Mech. Engrg., 193: 2667-2698.

Borja, R.I., Sama, K.M., Sanz, P.F., 2003. On the numerical integration of three-invariant elastoplastic constitutive models. Comput. Methods Appl. Mech. Engrg., 192: 1227-1258.

Brace, F.W., Kohlstedt, D.L., 1980. Limits on lithospheric stress imposed by laboratory experiments. J. Geophys. Res., 50: 6248-6252.

Braun, J., Sambridge, M., 1994. Dynamical Lagrangian Remeshing (DLR): A new algorithm for solving large strain deformation problems and its application to fault propagation folding. Earth Planet. Sci. Lett., 124: 211-220.

Brezzi, F., 1974. On the existence, uniqueness and approximation of saddle-point problems arising from Lagrange multipliers. RAIRO, 8: 129-151.

Buiter, S.J.H., Babeyko, A.Yu., Ellis, S., Gerya, T.V., Kaus, B.J.P., Kellner, A., Schreurs, G., Yamada, Y., 2006. The Numerical Sandbox: Comparison of model results for a shortening and an extension experiment, analogue and numerical modelling of crustal-scale processes. Geological Society, London, Special Publication, 253: 29-64.

Bunge, H.-P., Baumgardner, J.R., 1995. Mantle convection modeling on parallel virtual machines. Comput. Phys., 9: 207-215.

Christensen, U., Harder, H., 1991. 3-D Convection with variable viscosity. Geophys. J. Int. 104: 213-220.

Coulomb, C.A., 1773. Test on the applications of the rules of maxima and minima to some problems of statics related to architecture (in French). Mem. de Math. et de Phys., 7: 343-382.

Crisfield, M.A., 1983. An arc-length method including line searches and accelerations. Int. J. Numer. Meth. Engng., 19: 1269-1289.

Ekevid, T., Kettil, P., Wiberg, N.-E., 2004. Adaptive multigrid for finite element computations in plasticity. Comput. Struct., 82: 2413-2424.

Farhat, C., Lesoinne, M., Pierson, K., 2000. A scalable dual-primal domain decomposition method. Numer. Linear Algebra Appl., 7: 687-714.

Fish, J., Shek, K., 1999. Computational aspects of incrementally objective algorithms for large deformation plasticity. Int. J. Numer. Meth. Engng., 44: 839-851.

Flanagan, D.P., Belytschko, T., 1981. A uniform strain hexahedron and quadrilateral with orthogonal hourglass control. Int. J. Numer. Meth. Engng., 17: 679-706. 
Fullsack, P., 1995. An arbitrary Lagrangian-Eulerian formulation for creping flows and its application in tectonic models. Geophys. J. Int., 120: 1-23.

Geers, M.G.D., 1999. Enhanced solution control for physically and geometrically non-linear problems. Part I-the subplane control approach. Int. J. Numer. Meth. Engng., 46: 177-204.

George, A., 1973. Nested dissection of a regular finite element mesh. SIAM J. Numer. Analysis, 10: 345363.

Gerya, T.V., Yuen, D.A., 2007. Robust characteristics method for modelling multiphase visco-elastoplastic thermo-mechanical problems. Phys. Earth Planet. Inter., 163: 83-105.

Gupta, A., Karypis, G., Kumar, V., 1997. Highly scalable parallel algorithms for parse matrix factorization. IEEE Trans. Parallel Distrib. Syst. 8: 502-520.

Harlow, F., Welsh, J., 1965. Numerical calculation of time-dependant viscous incompressible flow of fluid with free surface. Phys. Fluids, 8: 2182-2189.

Hestenes, M.R., Stiefel, E., 1952. Methods of conjugate gradients for solving linear systems. J. Res. Nat. Bur. Stand., 49: 409-436.

Hirt, C.W., Amsden, A.A., Cook, J.L., 1974. An arbitrary Lagrangian-Eulerian computing method for all flow speeds. J. Comput. Phys., 14: 227-253.

Hughes, T.J.R., Winget, J., 1980. Finite rotation effects in numerical integration of rate constitutive equations arising in large-deformation analysis. Int. J. Numer. Meth. Engng., 15: 1862-1867.

Hughes, T.J.R., 1987. The Finite Element Method, Prentice-Hall, Englewood-Cliffs, New Jersey.

Kameyama, M., Yuen, D.A., Karato, S.-I., 1999. Thermal-mechanical effects of low-temperature plasticity (the Peierls mechanism) on the deformation of a viscoelastic shear zone. Earth Planet. Sci. Lett.. 168: 159-172.

Karato, S.-I., Riedel, M.R., Yuen, D.A., 2001. Rheological structure and deformation of subducted slabs in the mantle transition zone: implications for mantle circulation and deep earthquakes. Phys. Earth Planet. Inter., 127: 83-108.

Kaus, B.J.P., Podladchikov, Y.Y., 2006. Initiation of localized shear zones in viscoelastoplastic rocks. J. Geophys. Res., 111, B04412, doi: 10.1029/2005JB003652.

Ladyzhenskaya, O.A., 1969. The Mathematical Theory of Viscous Incompressible Flow, 2nd edn. Gordon \& Breach, New York.

Larsson, R., Runesson, K., 1996. Implicit integration and consistent linearization for yield criteria of the Mohr-Coulomb type. Mech. Cohes.-Frict. Mat., 1: 367-383.

Liu, W.K., Guo, Y., Tang, S., Belytschko, T., 1998. A multiple-quadrature eight-node hexahedral finite element for large deformation elastoplastic analysis. Comput. Methods Appl. Mech. Engrg., 154: 69132.

Malkus, D.S., Hughes, T.J.R., 1978. Mixed finite element methods - reduced and selective integration techniques: a unification of concept. Comput. Methods Appl. Mech. Engrg., 15: 63-81.

Moresi, L.N., Dufour, F., Muhlhaus, H., 2003. A Lagrangian integration point finite element method for large deformation modeling of viscoelastic geomaterials. J. Comput. Phys., 184: 476-497. 
Muhlhaus, H.-B., Aifantis, E.C., 1991. A variational principle for gradient plasticity. Int. J. Solids Struct. 28: $845-857$.

Muhlhaus, H.-B., Regenauer-Lieb, K., 2005. Towards a self-consistent plate mantle model that includes elasticity: simple benchmarks and application to basic modes of convection. Geophys. J. Int., 163: 788-800.

Muhlhaus, H.-B., Vardoulakis, I., 1987. The thickness of shear bands in granular materials. Geotechnique, 37: 271-283.

O’Neill, C., Moresi, L., Muller, D., Albert, R., Dufour, F., 2006. Ellipsis 3D: A particle-in-cell finiteelement hybrid code for modelling mantle convection and lithospheric deformation. Comput. Geosci., 32: 1769-1779.

Ortiz, M., Popov, E.P., 1985. Accuracy and stability of integration algorithms for elastoplastic constitutive relations. Int. J. Numer. Meth. Engng., 21: 1561-1576.

Perez-Foguet, A., Rodriguez-Ferran, A., Huerta, A., 2000. Numerical differentiation for local and global tangent operators in computational plasticity. Comput. Methods Appl. Mech. Engrg., 189: 277-296.

Petrunin, A., Sobolev, S.V., 2006. What controls thickness of sediments and lithospheric deformation at a pull-apart basin? Geology, 34: 389-392.

Poliakov, A.N., Cundall, P.A., Podladchikov, Y.Y., Lyakhovsky, V.A., 1993. An explicit inertial method for the simulation of the viscoelastic flow: an evaluation of elastic effects on diapiric flow in twoand three-layers models. In: D.B. Stone and S.K. Runcorn (Editors). Flow and creep in the Solar System: observations, modelling and theory, Kluwer Academic Publishers, 175-195.

Poliakov, A.N., Herrmann, H.J., 1994. Self-organized criticality of plastic shear bands in rocks. Geophys. Res. Lett., 21: 2143-2146.

Press, W.H., Teukolsky, S.A., Vetterling, W.T., Flannery, B.P., 2002. Numerical recipes in C++: the art of scientific computing, 2nd edn. Cambridge University Press, Cambridge.

Puso, M.A., Solberg, J., 2006. A stabilized nodally integrated tetrahedral. Int. J. Numer. Meth. Engng., 67: 841-867.

Rashid, M.M., 1993. Incremental kinematics for finite element applications. Int. J. Numer. Meth. Engng., 36: 3937-3956.

Rassineux, A., 1998. Generation and optimization of tetrahedral meshes by advancing front technique. Int. J. Numer. Meth. Engng., 41: 651-674.

Reese, S., 2003. On a consistent hourglass stabilization technique to treat large inelastic deformations and thermo-mechanical coupling in plane strain problems. Int. J. Numer. Meth. Engng., 57: 1095-1127.

Regenauer-Lieb, K., 2006. Water and Geodynamics. Rev. Miner. Geochem., 62: 451-473.

Regenauer-Lieb, K., Yuen, D.A. 2003. Modeling shear zones in geological and planetary sciences: solidand fluid-thermal-mechanical approaches. Earth Sci. Rev., 63: 295-349.

Regenauer-Lieb, K., Yuen, D.A. 2004. Positive feedback of interacting ductile faults from coupling of equation of state, rheology and thermal-mechanics. Phys. Earth Planet. Inter., 142: 113-135. 
Roscoe, K.H., 1970. The influence of strains in soil mechanics, 10th Rankine Lecture. Geotechnique, 20: 129-170.

Saad, Y., 1994. ILUT: a dual threshold incomplete ILU factorization. Numer. Linear Algebra Appl., 1: $387-402$.

Schmalholz, S.M., Podladchikov, Y.Y., Schmid, D.W., 2001. A spectral/finite difference method for simulating large deformations of heterogeneous, viscoelastic materials. Geophys. J. Int., 145, 199208.

Shampine, L.F., Watts, H.A., 1970. FZERO, a root-solving code. Report SC-TM-70-631, Sandia Laboratories.

Shephard, M.S., Georges, M.K., 1991. Automatic three-dimensional mesh generation by the finite octree technique. Int. J. Numer. Meth. Engng., 32: 709-749.

Shih, T.M., Tan, C.H., Hwang, B.C., 1989. Effects of grid staggering on numerical schemes. Int. J. Numer. Meth. Fluids, 9: 193-212.

Simo, J.C., Hughes, T.J.R., 2000. Computational Inelasticity, 2nd edn. Springer-Verlag, New York.

Simo, J.C., Taylor, R.L., 1985. Consistent tangent operators for rate-independent elastoplasticity. Comput. Methods Appl. Mech. Engrg., 48: 101-118.

Simo, J.C., Kennedy, J.G., Govindjee, S., 1988. Non-smooth multisurface plasticity and viscoplasticity. Loading/unloading conditions and numerical algorithms. Int. J. Numer. Meth. Engng., 26: 21612185 .

Slezkin, A., 1955. Dynamics of viscous incompressible fluid (in Russian). Gostekhizdat, Moscow.

Sloan, S.W., Booker, J.R., 1986. Removal of singularities in Tresca and Mohr-Coulomb yield functions. Commun. Appl. Numer. Meth., 2: 173-179.

Sobolev, S.V., Petrunin, A., Garfunkel, Z., Babeyko, A.Y., DESERT Group., 2005. Thermo-mechanical model of the Dead Sea transformation. Earth Planet. Sci. Lett., 238: 78-95.

Tackley, P.J., Xie, S., 2003. STAG3D: A code for modeling thermo-chemical multiphase convection in Earth's mantle. In: K.J. Bathe (Editor). Proceedings of the Second MIT Conference on Computational Fluid and Solid Mechanics. Elsevier B.V., Amsterdam, 1524-1527.

Trompert, R.A., Hansen, U., 1996. The application of a finite-volume multigrid method to 3-dimensional flow problems in a highly viscous fluid with a variable viscosity. Geophys. Astrophys. Fluid Dyn., 83: 261-291.

Vermeer, P.A., 1990. The orientation of shear bands in biaxial tests. Geotechnique, 40: 223-226.

Weinberg, R.B., Schmeling, H., 1992. Polydiapirs: multiwavelength gravity structures. J. Struct. Geol., 14: $425-436$.

Wilkins, M.L., 1964. Calculation of elastic-plastic flow. In: B. Alder (Editor). Methods in Computational Physics, Academic press, New York, 211-263.

Zienkiewicz, O.C., Taylor, R.L., 2000. The finite element method, 5th edn. Butterworth-Heinemann, Oxford. 
Zhong, S., Gurnis, M., 1996. Incorporation of fault-bound plates in three-dimensional models of mantle flow. Nature, 383: 245-247. 